\title{
Geometry of Centroaffine Surfaces in $\mathbb{R}^{5}$
}

\author{
Nathaniel BUSHEK ${ }^{\dagger}$ and Jeanne N. CLELLAND $\ddagger$ \\ $\dagger$ Department of Mathematics, UNC-Chapel Hill, \\ CB \#3250, Phillips Hall, Chapel Hill, NC 27599, USA \\ E-mail: bushek@unc.edu \\ $\ddagger$ Department of Mathematics, 395 UCB, University of Colorado, Boulder, CO 80309-0395, USA \\ E-mail: Jeanne.Clelland@colorado.edu
}

Received August 23, 2014, in final form December 26, 2014; Published online January 06, 2015

http://dx.doi.org/10.3842/SIGMA.2015.001

\begin{abstract}
We use Cartan's method of moving frames to compute a complete set of local invariants for nondegenerate, 2-dimensional centroaffine surfaces in $\mathbb{R}^{5} \backslash\{0\}$ with nondegenerate centroaffine metric. We then give a complete classification of all homogeneous centroaffine surfaces in this class.
\end{abstract}

Key words: centroaffine geometry; Cartan's method of moving frames

2010 Mathematics Subject Classification: 53A15; 58A15

\section{Introduction}

An immersion $\bar{f}: M \rightarrow \mathbb{R}^{n} \backslash\{0\}$ is called a centroaffine immersion (cf. Definition 2.1) if the position vector $\bar{f}(x)$ is transversal to the tangent space $\bar{f}_{*}\left(T_{x} M\right)$ for all $x \in M$. Centroaffine geometry is the study of those properties of centroaffine immersions that are invariant under the action of the centroaffine group $\mathrm{GL}(n, \mathbb{R})$ on $\mathbb{R}^{n} \backslash\{0\}$. Much attention has been given to the study of centroaffine curves and hypersurfaces $[5,8,9,10,11,17,18,20]$, and more recently to the study of centroaffine immersions of codimension $2[3,4,13,15,16,19,22,23]$. In [21], applications of centroaffine geometry to the problem of feedback equivalence in control theory are discussed.

In this paper, we consider the case of a 2 -dimensional centroaffine surface in $\mathbb{R}^{5} \backslash\{0\}$. We will use Cartan's method of moving frames to construct a complete set of local invariants for a large class of such surfaces under certain nondegeneracy assumptions (cf. Definition 3.1, Assumption 3.3). In addition, we give a complete classification of the homogeneous centroaffine surfaces in this class - i.e., those that admit a 3-dimensional Lie group of symmetries that acts transitively on an adapted frame bundle canonically associated to the surface (cf. Definition 6.1). Our primary results are Theorems 4.3 and 5.3, which describe local invariants for centroaffine surfaces with definite and indefinite centroaffine metrics, respectively, and Theorem 6.6, which describes the homogeneous examples.

The paper is organized as follows. In Section 2, we introduce the basic concepts of centroaffine geometry and centroaffine surfaces in $\mathbb{R}^{5} \backslash\{0\}$, including the centroaffine frame bundle and the Maurer-Cartan forms. In Section 3, we begin the method of moving frames and identify firstorder invariants for centroaffine surfaces. Based on these invariants, nondegenerate surfaces may be locally classified as "space-like", "time-like", or "null". In Sections 4 and 5, we continue the method of moving frames for the space-like and time-like cases, respectively. (We do not consider the null case here; it may be explored in a future paper.) Finally, in Section 6 we classify the homogeneous examples in both the space-like and time-like cases. 


\section{Centroaffine surfaces in $\mathbb{R}^{5}$, adapted frames, and Maurer-Cartan forms}

Five-dimensional centroaffine space is the manifold $\mathbb{R}^{5} \backslash\{0\}$, equipped with a natural GL(5, $\left.\mathbb{R}\right)$ action. Specifically, $\mathrm{GL}(5, \mathbb{R})$ acts on $\mathbb{R}^{5} \backslash\{0\}$ by left multiplication: for $\mathbf{x} \in \mathbb{R}^{5} \backslash\{0\}, g \in$ $\mathrm{GL}(5, \mathbb{R})$, we have

$$
g \cdot \mathbf{x}=g \mathbf{x}
$$

The group $\mathrm{GL}(5, \mathbb{R})$ may be regarded as a principal bundle over $\mathbb{R}^{5} \backslash\{0\}$ : write an arbitrary element $g \in \mathrm{GL}(5, \mathbb{R})$ as

$$
g=\left[\begin{array}{lllll}
\mathbf{e}_{0} & \mathbf{e}_{1} & \mathbf{e}_{2} & \mathbf{e}_{3} & \mathbf{e}_{4}
\end{array}\right]
$$

where $\mathbf{e}_{0}, \ldots, \mathbf{e}_{4} \in \mathbb{R}^{5} \backslash\{0\}$ are linearly independent column vectors. Then define the bundle $\operatorname{map} \pi: \operatorname{GL}(5, \mathbb{R}) \rightarrow \mathbb{R}^{5} \backslash\{0\}$ by

$$
\pi\left(\left[\begin{array}{lllll}
\mathbf{e}_{0} & \mathbf{e}_{1} & \mathbf{e}_{2} & \mathbf{e}_{3} & \mathbf{e}_{4}
\end{array}\right]\right)=\mathbf{e}_{0} .
$$

The fiber group $H$ is isomorphic to the stabilizer of the point

$$
\left[\begin{array}{lllll}
1 & 0 & 0 & 0 & 0
\end{array}\right]^{\mathrm{T}} \in \mathbb{R}^{5} \backslash\{0\},
$$

and this construction endows the manifold $\mathbb{R}^{5} \backslash\{0\}$ with the structure of the homogeneous space $\mathrm{GL}(5, \mathbb{R}) / H$.

We also think of the bundle $\pi: \operatorname{GL}(5, \mathbb{R}) \rightarrow \mathbb{R}^{5} \backslash\{0\}$ as the centroaffine frame bundle $\mathcal{F}$ over $\mathbb{R}^{5} \backslash\{0\}$. For each point $\mathbf{e}_{0} \in \mathbb{R}^{5} \backslash\{0\}$, the fiber over $\mathbf{e}_{0}$ consists of all frames $\left(\mathbf{e}_{0}, \mathbf{e}_{1}, \mathbf{e}_{2}, \mathbf{e}_{3}, \mathbf{e}_{4}\right)$ for the tangent space $T_{\mathbf{e}_{0}}\left(\mathbb{R}^{5} \backslash\{0\}\right)$ - i.e., all frames for which the first vector in the frame is equal to the position vector.

The Maurer-Cartan forms $\omega_{j}^{i}$ on $\mathcal{F}$ are defined by the equations

$$
d \mathbf{e}_{i}=\mathbf{e}_{j} \omega_{i}^{j}, \quad 0 \leq i, j \leq 4,
$$

and they satisfy the Cartan structure equations

$$
d \omega_{j}^{i}=-\omega_{k}^{i} \wedge \omega_{j}^{k}
$$

(For details, see [7, p. 18] or [1].)

We are interested in the geometry of 2-dimensional immersions $\bar{f}: M^{2} \rightarrow \mathbb{R}^{5} \backslash\{0\}$; we will use Cartan's method of moving frames to compute local invariants for such immersions under the action of $\operatorname{GL}(5, \mathbb{R})$.

Definition 2.1. An immersion $\bar{f}$ of a 2-dimensional manifold $M$ into $\mathbb{R}^{5} \backslash\{0\}$ is called a centroaffine immersion if the position vector $\bar{f}(x)$ is transversal to the tangent space $\bar{f}_{*}\left(T_{x} M\right)$ for all $x \in M$. The image $\Sigma=\bar{f}(M)$ is called a centroaffine surface in $\mathbb{R}^{5} \backslash\{0\}$.

In order to begin the method of moving frames, consider the induced bundle of centroaffine frames along $\Sigma=\bar{f}(M)$; this is simply the pullback bundle $\mathcal{F}_{0}=\bar{f}^{*} \mathcal{F}$ over $M$. A centroaffine frame field along $\Sigma$ is a section of $\mathcal{F}_{0}$ - i.e., a smooth map $f: M \rightarrow \operatorname{GL}(5, \mathbb{R})$ such that $\pi \circ f=\bar{f}$. Throughout the remainder of this paper, we will consider the pullbacks of the Maurer-Cartan forms on $\mathcal{F}$ to $M$ via such sections $f$, and we will suppress the pullback notation.

We will gradually adapt our choice of centroaffine frame fields based on the geometry of $\Sigma$. For our first adaptation, consider the subbundle $\mathcal{F}_{1} \subset \mathcal{F}_{0}$ consisting of all frames for which 
$\left(\mathbf{e}_{1}(x), \mathbf{e}_{2}(x)\right)$ span the tangent space $T_{\bar{f}(x)} \Sigma$ for each $x \in M$. A section $f: M \rightarrow \mathcal{F}_{1}$ will be called a 1-adapted frame field along $\Sigma$. Any two 1-adapted frames $\left(\mathbf{e}_{0}, \ldots, \mathbf{e}_{4}\right),\left(\tilde{\mathbf{e}}_{0}, \ldots, \tilde{\mathbf{e}}_{4}\right)$ based at the same point $x \in M$ are related by a transformation of the form

$$
\left[\begin{array}{lllll}
\tilde{\mathbf{e}}_{0} & \tilde{\mathbf{e}}_{1} & \tilde{\mathbf{e}}_{2} & \tilde{\mathbf{e}}_{3} & \tilde{\mathbf{e}}_{4}
\end{array}\right]=\left[\begin{array}{lllll}
\mathbf{e}_{0} & \mathbf{e}_{1} & \mathbf{e}_{2} & \mathbf{e}_{3} & \mathbf{e}_{4}
\end{array}\right]\left[\begin{array}{ccccc}
1 & 0 & 0 & r_{03} & r_{04} \\
0 & a_{11} & a_{12} & r_{13} & r_{14} \\
0 & a_{21} & a_{22} & r_{23} & r_{24} \\
0 & 0 & 0 & b_{33} & b_{34} \\
0 & 0 & 0 & b_{43} & b_{44}
\end{array}\right],
$$

where the $2 \times 2$ submatrices

$$
A=\left[\begin{array}{ll}
a_{11} & a_{12} \\
a_{21} & a_{22}
\end{array}\right], \quad B=\left[\begin{array}{ll}
b_{33} & b_{34} \\
b_{43} & b_{44}
\end{array}\right]
$$

are elements of $\operatorname{GL}(2, \mathbb{R})$. We will denote the group of all matrices of the form in (2.4) by $G_{1}$; then the bundle $\mathcal{F}_{1}$ is a principal bundle over $M$ with fiber group $G_{1}$.

If $f, \tilde{f}: M \rightarrow \mathcal{F}_{1}$ are two 1-adapted frame fields along $\Sigma$, then $f, \tilde{f}$ are related by the equation

$$
\tilde{f}(x)=f(x) \cdot g(x)
$$

for some smooth function $g: M \rightarrow G_{1}$, as in equation (2.4). Then the corresponding $\mathfrak{g l}(5, \mathbb{R})$ valued Maurer-Cartan forms $\Omega=\left[\omega_{j}^{i}\right], \tilde{\Omega}=\left[\tilde{\omega}_{j}^{i}\right]$ on $M$ are related as follows:

$$
\tilde{\Omega}=g^{-1} d g+g^{-1} \Omega g .
$$

\section{Reduction of the structure group and first-order invariants}

Now consider the pullbacks of the Maurer-Cartan forms to $M$ via a 1-adapted frame field $f$. From equation (2.2) for $d \mathbf{e}_{0}$ and the fact that the image of $d \mathbf{e}_{0}$ is spanned by $\mathbf{e}_{1}$ and $\mathbf{e}_{2}$, we have

$$
\omega_{0}^{0}=\omega_{0}^{3}=\omega_{0}^{4}=0 .
$$

Moreover, the 1-forms $\omega_{0}^{1}, \omega_{0}^{2}$ are semi-basic for the projection $\pi: \mathcal{F}_{1} \rightarrow M$; in fact, they form a basis for the semi-basic 1 -forms on $\mathcal{F}_{1}$.

Differentiating equations (3.1) yields:

$$
\begin{aligned}
& 0=d \omega_{0}^{0}=-\left(\omega_{1}^{0} \wedge \omega_{0}^{1}+\omega_{2}^{0} \wedge \omega_{0}^{2}\right), \\
& 0=d \omega_{0}^{3}=-\left(\omega_{1}^{3} \wedge \omega_{0}^{1}+\omega_{2}^{3} \wedge \omega_{0}^{2}\right), \\
& 0=d \omega_{0}^{4}=-\left(\omega_{1}^{4} \wedge \omega_{0}^{1}+\omega_{2}^{4} \wedge \omega_{0}^{2}\right) .
\end{aligned}
$$

Cartan's lemma (see, e.g., [7] or [1]) then implies that there exist functions $h_{i j}^{k}=h_{j i}^{k}, k=0,3,4$, on $M$ such that

$$
\left[\begin{array}{c}
\omega_{1}^{k} \\
\omega_{2}^{k}
\end{array}\right]=\left[\begin{array}{ll}
h_{11}^{k} & h_{12}^{k} \\
h_{12}^{k} & h_{22}^{k}
\end{array}\right]\left[\begin{array}{l}
\omega_{0}^{1} \\
\omega_{0}^{2}
\end{array}\right] .
$$

For simplicity of notation, let $h^{k}$ denote the matrix

$$
h^{k}=\left[\begin{array}{ll}
h_{11}^{k} & h_{12}^{k} \\
h_{12}^{k} & h_{22}^{k}
\end{array}\right], \quad k=0,3,4 .
$$


If $f, \tilde{f}: M \rightarrow \mathcal{F}_{1}$ are two 1-adapted frame fields related by a transformation of the form (2.4), then we can use equation (2.5) to determine how the corresponding matrices $h^{k}, \tilde{h}^{k}$ are related. First, it follows from the fact that $\tilde{\mathbf{e}}_{0}=\mathbf{e}_{0}$ that

$$
d \tilde{\mathbf{e}}_{0}=\left[\begin{array}{ll}
\tilde{\mathbf{e}}_{1} & \tilde{\mathbf{e}}_{2}
\end{array}\right]\left[\begin{array}{c}
\tilde{\omega}_{0}^{1} \\
\tilde{\omega}_{0}^{2}
\end{array}\right]=\left[\begin{array}{ll}
\mathbf{e}_{1} & \mathbf{e}_{2}
\end{array}\right]\left[\begin{array}{c}
\omega_{0}^{1} \\
\omega_{0}^{2}
\end{array}\right]=d \mathbf{e}_{0} .
$$

Then, since we have

$$
\left[\begin{array}{ll}
\tilde{\mathbf{e}}_{1} & \tilde{\mathbf{e}}_{2}
\end{array}\right]=\left[\begin{array}{ll}
\mathbf{e}_{1} & \mathbf{e}_{2}
\end{array}\right] A,
$$

we must have

$$
\left[\begin{array}{c}
\tilde{\omega}_{0}^{1} \\
\tilde{\omega}_{0}^{2}
\end{array}\right]=A^{-1}\left[\begin{array}{c}
\omega_{0}^{1} \\
\omega_{0}^{2}
\end{array}\right] .
$$

Similar considerations show that

$$
\begin{aligned}
& {\left[\begin{array}{l}
\tilde{\omega}_{1}^{3} \\
\tilde{\omega}_{2}^{3}
\end{array}\right]=\frac{1}{(\operatorname{det} B)} A^{\mathrm{T}}\left(b_{44}\left[\begin{array}{c}
\omega_{1}^{3} \\
\omega_{2}^{3}
\end{array}\right]-b_{34}\left[\begin{array}{c}
\omega_{1}^{4} \\
\omega_{2}^{4}
\end{array}\right]\right),} \\
& {\left[\begin{array}{c}
\tilde{\omega}_{1}^{4} \\
\tilde{\omega}_{2}^{4}
\end{array}\right]=\frac{1}{(\operatorname{det} B)} A^{\mathrm{T}}\left(-b_{43}\left[\begin{array}{c}
\omega_{1}^{3} \\
\omega_{2}^{3}
\end{array}\right]+b_{33}\left[\begin{array}{c}
\omega_{1}^{4} \\
\omega_{2}^{4}
\end{array}\right]\right),} \\
& {\left[\begin{array}{c}
\tilde{\omega}_{1}^{0} \\
\tilde{\omega}_{2}^{0}
\end{array}\right]=A^{\mathrm{T}}\left[\begin{array}{c}
\omega_{1}^{0} \\
\omega_{2}^{0}
\end{array}\right]-r_{03}\left[\begin{array}{c}
\tilde{\omega}_{1}^{3} \\
\tilde{\omega}_{2}^{3}
\end{array}\right]-r_{04}\left[\begin{array}{c}
\tilde{\omega}_{1}^{4} \\
\tilde{\omega}_{2}^{4}
\end{array}\right] .}
\end{aligned}
$$

Together, equations (3.2), (3.3) imply that

$$
\begin{aligned}
& \tilde{h}^{3}=\frac{1}{(\operatorname{det} B)} A^{\mathrm{T}}\left(b_{44} h^{3}-b_{34} h^{4}\right) A, \quad \tilde{h}^{4}=\frac{1}{(\operatorname{det} B)} A^{\mathrm{T}}\left(-b_{43} h^{3}+b_{33} h^{4}\right) A, \\
& \tilde{h}^{0}=A^{\mathrm{T}} h^{0} A-r_{03} \tilde{h}^{3}-r_{04} \tilde{h}^{4} .
\end{aligned}
$$

Definition 3.1. A centroaffine surface $\Sigma=\bar{f}(M)$ will be called nondegenerate if the matrices $h^{0}, h^{3}, h^{4}$ are linearly independent in $\operatorname{Sym}^{2}(\mathbb{R})$ at every point of $M$.

Henceforth, we assume that $\Sigma$ is nondegenerate; from the group action (3.4) it is clear that this definition is independent of the choice of 1-adapted frame field $f: M \rightarrow \mathcal{F}_{1}$ along $\Sigma$.

The next step is to use the group action (3.4) to find normal forms for the matrices $h^{0}, h^{3}, h^{4}$. First consider the action on $h^{3}, h^{4}$ : it can be written as the composition of two separate actions by the matrices $A, B \in \mathrm{GL}(2, \mathbb{R})$ :

$$
\begin{aligned}
& A \cdot\left(h^{3}, h^{4}\right)=\left(A^{\mathrm{T}} h^{3} A, A^{\mathrm{T}} h^{4} A\right), \\
& B \cdot\left(h^{3}, h^{4}\right)=\left(\frac{1}{(\operatorname{det} B)}\left(b_{44} h^{3}-b_{34} h^{4}\right), \frac{1}{(\operatorname{det} B)}\left(-b_{43} h^{3}+b_{33} h^{4}\right)\right) .
\end{aligned}
$$

If we let $P$ denote the 2 -dimensional subspace of $\operatorname{Sym}^{2}(\mathbb{R})$ spanned by $\left(h^{3}, h^{4}\right)$, then we see that the action by $B$ preserves $P$, while $A$ acts on $P$ via

$$
A \cdot P=A^{\mathrm{T}} P A .
$$

In order to understand the action (3.5), consider the related action

$$
A \cdot h=A^{\mathrm{T}} h A
$$


on $\operatorname{Sym}^{2}(\mathbb{R})$. It is shown in $[12$, p. 115] that this action preserves the indefinite quadratic form

$$
Q(h)=-\operatorname{det}(h)
$$

up to a scale factor. More precisely, $Q$ gives $\operatorname{Sym}^{2}(\mathbb{R})$ the structure of the Minkowski space $\mathbb{R}^{2,1}$, and the action $(3.6)$ gives a representation of $\mathrm{GL}(2, \mathbb{R})$ as the group $\mathrm{CSO}^{+}(2,1)$ of orientationpreserving, orthochronous, conformal Minkowski transformations of $\mathbb{R}^{2,1}$. This action has precisely 6 orbits, represented by the matrices

$$
\left[\begin{array}{ll}
0 & 0 \\
0 & 0
\end{array}\right], \quad\left[\begin{array}{ll}
1 & 0 \\
0 & 0
\end{array}\right], \quad\left[\begin{array}{cc}
-1 & 0 \\
0 & 0
\end{array}\right], \quad\left[\begin{array}{ll}
1 & 0 \\
0 & 1
\end{array}\right], \quad\left[\begin{array}{cc}
1 & 0 \\
0 & -1
\end{array}\right], \quad\left[\begin{array}{cc}
-1 & 0 \\
0 & -1
\end{array}\right] .
$$

In terms of the Minkowski metric on $\operatorname{Sym}^{2}(\mathbb{R})$ determined by $Q$, the second and third matrices in (3.8) represent the two orbits of null vectors (oriented in opposite time directions); the fourth and fifth represent the two orbits of time-like vectors (oriented in opposite time directions), while the sixth represents the single orbit of space-like vectors.

The action (3.6) induces the action (3.5) on the Grassmannian of 2-planes in $\operatorname{Sym}^{2}(\mathbb{R})$, which corresponds to the action of $\mathrm{CSO}^{+}(2,1)$ on planes in $\mathbb{R}^{2,1}$. This action has precisely 3 orbits, consisting of planes that are space-like, time-like, or null with respect to the quadratic form (3.7). These orbits are represented by the 2-planes

$$
\begin{aligned}
& P_{1}=\operatorname{span}\left(\left[\begin{array}{cc}
1 & 0 \\
0 & -1
\end{array}\right],\left[\begin{array}{cc}
0 & 1 \\
1 & 0
\end{array}\right]\right) \quad \text { (space-like) } \\
& P_{2}=\operatorname{span}\left(\left[\begin{array}{ll}
1 & 0 \\
0 & 1
\end{array}\right],\left[\begin{array}{cc}
1 & 0 \\
0 & -1
\end{array}\right]\right) \quad \text { (time-like) } \\
& P_{3}=\operatorname{span}\left(\left[\begin{array}{ll}
1 & 0 \\
0 & 0
\end{array}\right],\left[\begin{array}{ll}
0 & 1 \\
1 & 0
\end{array}\right]\right) \quad \text { (null) }
\end{aligned}
$$

The type of the plane $P$ spanned by $\left(h^{3}, h^{4}\right)$ (space-like, time-like, or null) is preserved by the group action (3.4); thus the type of $P$ at any point $x \in M$ is well-defined, independent of the choice of 1-adapted frame field $f: M \rightarrow \mathcal{F}_{1}$.

Remark 3.2. We note that similar quadratic forms $h_{3}, h_{4}$ were obtained by Nomizu and Vrancken in their study of affine surfaces in $\mathbb{R}^{4}$; see [14].

At this point, the method of moving frames dictates that we divide into cases based on the type of $P$. In order to proceed, we make the following assumption:

Assumption 3.3. Assume that $\Sigma$ has constant type - i.e., that the type of $P$ is the same at every point $x \in M$.

In this paper we will consider only the space-like and time-like cases; the null case is considerably more complicated and may be explored in a future paper.

\section{The space-like case}

First, suppose that the plane $P$ spanned by $\left(h^{3}, h^{4}\right)$ is space-like at every point of $M$. According to the group action (3.5), we can find a 1-adapted frame field along $\Sigma$ for which $P=P_{1}$, as in equation (3.9). Furthermore, we can then use the action by $B$ to find a 1-adapted frame field along $\Sigma$ for which

$$
h^{3}=\left[\begin{array}{cc}
1 & 0 \\
0 & -1
\end{array}\right], \quad h^{4}=\left[\begin{array}{ll}
0 & 1 \\
1 & 0
\end{array}\right] .
$$


The next step is to determine the subgroup of $G_{1}$ that preserves the conditions (4.1). To this end, first note that the plane $P_{1}$ spanned by the matrices (4.1) consists precisely of the trace-free matrices in $\operatorname{Sym}^{2}(\mathbb{R})$. A straightforward computation shows that, with $h^{3}, h^{4}$ as in (4.1),

$$
\operatorname{tr}\left(A^{\mathrm{T}} h^{3} A\right)=a_{11}^{2}+a_{12}^{2}-a_{21}^{2}-a_{22}^{2}, \quad \operatorname{tr}\left(A^{\mathrm{T}} h^{4} A\right)=2\left(a_{11} a_{21}+a_{12} a_{22}\right) .
$$

Therefore, the action (3.5) preserves $P_{1}$ if and only if

$$
a_{11}^{2}+a_{12}^{2}-a_{21}^{2}-a_{22}^{2}=a_{11} a_{21}+a_{12} a_{22}=0,
$$

which is true if and only if

$$
A=\lambda A_{0}
$$

for some $\lambda \in \mathbb{R}^{*}, A_{0} \in \mathrm{O}(2, \mathbb{R})$. Substituting this condition into equations (3.4) and imposing the conditions $\tilde{h}^{3}=h^{3}, \tilde{h}^{4}=h^{4}$ then yields

$$
B=\lambda^{2}\left(A_{0}\right)^{2} .
$$

For simplicity, we will restrict to transformations with $A_{0} \in \mathrm{SO}(2, \mathbb{R}), \lambda>0$; this has the advantage of producing a frame bundle whose fiber is a connected Lie group. Thus we will assume that

$$
A=\left[\begin{array}{cc}
\lambda \cos (\theta) & -\lambda \sin (\theta) \\
\lambda \sin (\theta) & \lambda \cos (\theta)
\end{array}\right], \quad B=\left[\begin{array}{cc}
\lambda^{2} \cos (2 \theta) & -\lambda^{2} \sin (2 \theta) \\
\lambda^{2} \sin (2 \theta) & \lambda^{2} \cos (2 \theta)
\end{array}\right]
$$

where $\lambda>0, \theta \in \mathbb{R}$.

Next, consider the effect of the action (3.4) on $h^{0}$. With $A=I_{2}$ and $r_{03}, r_{04}$ chosen appropriately, we can add any linear combination of $h^{3}, h^{4}$ to $h^{0}$. Thus we can find a 1-adapted frame field for which $h^{0}$ is a multiple (nonzero by the nondegeneracy assumption) of the identity matrix $I_{2}$. Then under the action (3.4) with $A, B$ as in (4.2), we have

$$
\tilde{h}^{0}=\lambda^{2} h^{0}+\left[\begin{array}{cc}
-r_{03} & -r_{04} \\
-r_{04} & r_{03}
\end{array}\right] .
$$

Therefore, we can find a 1-adapted frame field along $\Sigma$ satisfying the additional condition that $h^{0}= \pm I_{2}$, and this condition is preserved by transformations of the form (3.4) with $A, B$ as in (4.2), $\lambda=1$, and and $r_{03}=r_{04}=0$.

Definition 4.1. Let $\Sigma=\bar{f}(M)$ be a nondegenerate, space-like centroaffine surface in $\mathbb{R}^{5} \backslash\{0\}$. A 1-adapted frame field $f: M \rightarrow \mathcal{F}_{1}$ will be called 2-adapted if it satisfies the conditions

$$
h^{3}=\left[\begin{array}{cc}
1 & 0 \\
0 & -1
\end{array}\right], \quad h^{4}=\left[\begin{array}{ll}
0 & 1 \\
1 & 0
\end{array}\right], \quad h^{0}=\left[\begin{array}{ll}
\epsilon & 0 \\
0 & \epsilon
\end{array}\right],
$$

with $\epsilon= \pm 1$, at every point of $M$.

Any two 2-adapted frames $\left(\mathbf{e}_{0}, \ldots, \mathbf{e}_{4}\right),\left(\tilde{\mathbf{e}}_{0}, \ldots, \tilde{\mathbf{e}}_{4}\right)$ based at the same point $x \in M$ are related by a transformation of the form

$$
\begin{aligned}
& {\left[\begin{array}{lllll}
\tilde{\mathbf{e}}_{0} & \tilde{\mathbf{e}}_{1} & \tilde{\mathbf{e}}_{2} & \tilde{\mathbf{e}}_{3} & \tilde{\mathbf{e}}_{4}
\end{array}\right]} \\
& =\left[\begin{array}{lllll}
\mathbf{e}_{0} & \mathbf{e}_{1} & \mathbf{e}_{2} & \mathbf{e}_{3} & \mathbf{e}_{4}
\end{array}\right]\left[\begin{array}{ccccc}
1 & 0 & 0 & 0 & 0 \\
0 & \cos (\theta) & -\sin (\theta) & r_{13} & r_{14} \\
0 & \sin (\theta) & \cos (\theta) & r_{23} & r_{24} \\
0 & 0 & 0 & \cos (2 \theta) & -\sin (2 \theta) \\
0 & 0 & 0 & \sin (2 \theta) & \cos (2 \theta)
\end{array}\right] .
\end{aligned}
$$


We will denote the group of all matrices of the form in (4.4) by $G_{2}$; then the 2-adapted frame fields along $\Sigma$ are the smooth sections of a principal bundle $\mathcal{F}_{2} \subset \mathcal{F}_{1}$ over $M$ with fiber group $G_{2}$.

The equations (4.3) are equivalent to the condition that the Maurer-Cartan forms associated to a 2 -adapted frame field satisfy the conditions

$$
\omega_{1}^{3}=\omega_{0}^{1}, \quad \omega_{2}^{3}=-\omega_{0}^{2}, \quad \omega_{1}^{4}=\omega_{0}^{2}, \quad \omega_{2}^{4}=\omega_{0}^{1}, \quad \omega_{1}^{0}=\epsilon \omega_{0}^{1}, \quad \omega_{2}^{0}=\epsilon \omega_{0}^{2} .
$$

Differentiating equations (4.5) yields

$$
\begin{aligned}
& \left(2 \omega_{1}^{1}-\omega_{3}^{3}\right) \wedge \omega_{0}^{1}+\left(\omega_{2}^{1}-\omega_{1}^{2}-\omega_{4}^{3}\right) \wedge \omega_{0}^{2}=0, \\
& \left(\omega_{2}^{1}-\omega_{1}^{2}-\omega_{4}^{3}\right) \wedge \omega_{0}^{1}+\left(\omega_{3}^{3}-2 \omega_{2}^{2}\right) \wedge \omega_{0}^{2}=0, \\
& \left(2 \omega_{1}^{2}-\omega_{3}^{4}\right) \wedge \omega_{0}^{1}+\left(\omega_{1}^{1}+\omega_{2}^{2}-\omega_{4}^{4}\right) \wedge \omega_{0}^{2}=0, \\
& \left(\omega_{1}^{1}+\omega_{2}^{2}-\omega_{4}^{4}\right) \wedge \omega_{0}^{1}+\left(2 \omega_{2}^{1}+\omega_{3}^{4}\right) \wedge \omega_{0}^{2}=0, \\
& \left(2 \epsilon \omega_{1}^{1}-\omega_{3}^{0}\right) \wedge \omega_{0}^{1}+\left(\epsilon \omega_{2}^{1}+\epsilon \omega_{1}^{2}-\omega_{4}^{0}\right) \wedge \omega_{0}^{2}=0, \\
& \left(\epsilon \omega_{2}^{1}+\epsilon \omega_{1}^{2}-\omega_{4}^{0}\right) \wedge \omega_{0}^{1}+\left(2 \epsilon \omega_{2}^{2}+\omega_{3}^{0}\right) \wedge \omega_{0}^{2}=0 .
\end{aligned}
$$

Applying Cartan's lemma to these equations shows that there exists a 1-form $\alpha$ and functions $h_{j k}^{i}$ on $\mathcal{F}_{2}$ such that

$$
\begin{array}{ll}
\omega_{3}^{0}=h_{31}^{0} \omega_{0}^{1}+h_{32}^{0} \omega_{0}^{2}, & \omega_{4}^{0}=h_{41}^{0} \omega_{0}^{1}+h_{42}^{0} \omega_{0}^{2}, \\
\omega_{1}^{1}=h_{11}^{1} \omega_{0}^{1}+h_{12}^{1} \omega_{0}^{2}, & \omega_{2}^{1}=\alpha+h_{21}^{1} \omega_{0}^{1}+h_{22}^{1} \omega_{0}^{2}, \\
\omega_{1}^{2}=-\alpha+h_{21}^{1} \omega_{0}^{1}+h_{22}^{1} \omega_{0}^{2}, & \omega_{2}^{2}=h_{21}^{2} \omega_{0}^{1}+h_{22}^{2} \omega_{0}^{2}, \\
\omega_{3}^{3}=h_{31}^{3} \omega_{0}^{1}+h_{32}^{3} \omega_{0}^{2}, & \omega_{4}^{3}=2 \alpha+h_{41}^{3} \omega_{0}^{1}+h_{42}^{3} \omega_{0}^{2}, \\
\omega_{3}^{4}=-2 \alpha+h_{31}^{4} \omega_{0}^{1}+h_{32}^{4} \omega_{0}^{2}, & \omega_{4}^{4}=h_{41}^{4} \omega_{0}^{1}+h_{42}^{4} \omega_{0}^{2} .
\end{array}
$$

Moreover, the functions $h_{j k}^{i}$ satisfy the relations

$$
\begin{array}{ll}
2 h_{12}^{1}-h_{32}^{3}+h_{41}^{3}=0, & 2 h_{21}^{2}-h_{31}^{3}-h_{42}^{3}=0, \\
h_{11}^{1}-2 h_{22}^{1}+h_{21}^{2}+h_{32}^{4}-h_{41}^{4}=0, & h_{12}^{1}-2 h_{21}^{1}+h_{22}^{2}-h_{31}^{4}-h_{42}^{4}=0, \\
h_{32}^{0}-h_{41}^{0}+2 \epsilon\left(h_{21}^{1}-h_{12}^{1}\right)=0, & h_{31}^{0}+h_{42}^{0}+2 \epsilon\left(h_{21}^{2}-h_{22}^{1}\right)=0 .
\end{array}
$$

If $f, \tilde{f}: M \rightarrow \mathcal{F}_{2}$ are two 2-adapted frame fields related by a transformation of the form (4.4), then we can once again use equation (2.5) to determine how the corresponding functions $h_{j k}^{i}, \tilde{h}_{j k}^{i}$ are related. Some of these relationships are more complicated than others; the most straightforward to compute are those corresponding to the forms $\tilde{\omega}_{3}^{0}, \tilde{\omega}_{4}^{0}$. These forms appear as the coefficients of $\tilde{\mathbf{e}}_{0}=\mathbf{e}_{0}$ in the equations (2.2) for $d \tilde{\mathbf{e}}_{3}, d \tilde{\mathbf{e}}_{4}$. By applying equations (4.4) and (4.6), one can show that

$$
\begin{aligned}
& {\left[\begin{array}{l}
\tilde{h}_{31}^{0} \\
\tilde{h}_{41}^{0}
\end{array}\right]=\left[\begin{array}{cc}
\cos (2 \theta) & \sin (2 \theta) \\
-\sin (2 \theta) & \cos (2 \theta)
\end{array}\right]\left[\begin{array}{l}
h_{31}^{0} \\
h_{41}^{0}
\end{array}\right]+\epsilon\left[\begin{array}{l}
r_{13} \\
r_{14}
\end{array}\right],} \\
& {\left[\begin{array}{l}
\tilde{h}_{32}^{0} \\
\tilde{h}_{42}^{0}
\end{array}\right]=\left[\begin{array}{cc}
\cos (2 \theta) & \sin (2 \theta) \\
-\sin (2 \theta) & \cos (2 \theta)
\end{array}\right]\left[\begin{array}{l}
h_{32}^{0} \\
h_{42}^{0}
\end{array}\right]+\epsilon\left[\begin{array}{l}
r_{23} \\
r_{24}
\end{array}\right] .}
\end{aligned}
$$

Thus we can find a 2-adapted frame field along $\Sigma$ satisfying the conditions that

$$
h_{31}^{0}=h_{32}^{0}=h_{41}^{0}=h_{42}^{0}=0,
$$

and these conditions are preserved by transformations of the form (4.4) with $r_{13}=r_{14}=r_{23}=$ $r_{24}=0$. 
Definition 4.2. Let $\Sigma=\bar{f}(M)$ be a nondegenerate, space-like centroaffine surface in $\mathbb{R}^{5} \backslash\{0\}$. A 2-adapted frame field $f: M \rightarrow \mathcal{F}_{2}$ will be called 3-adapted if it satisfies the conditions

$$
h_{31}^{0}=h_{32}^{0}=h_{41}^{0}=h_{42}^{0}=0
$$

at every point of $M$.

Any two 3 -adapted frames $\left(\mathbf{e}_{0}, \ldots, \mathbf{e}_{4}\right),\left(\tilde{\mathbf{e}}_{0}, \ldots, \tilde{\mathbf{e}}_{4}\right)$ based at the same point $x \in M$ are related by a transformation of the form

$$
\begin{aligned}
& {\left[\begin{array}{lllll}
\tilde{\mathbf{e}}_{0} & \tilde{\mathbf{e}}_{1} & \tilde{\mathbf{e}}_{2} & \tilde{\mathbf{e}}_{3} & \tilde{\mathbf{e}}_{4}
\end{array}\right]} \\
& =\left[\begin{array}{lllll}
\mathbf{e}_{0} & \mathbf{e}_{1} & \mathbf{e}_{2} & \mathbf{e}_{3} & \mathbf{e}_{4}
\end{array}\right]\left[\begin{array}{ccccc}
1 & 0 & 0 & 0 & 0 \\
0 & \cos (\theta) & -\sin (\theta) & 0 & 0 \\
0 & \sin (\theta) & \cos (\theta) & 0 & 0 \\
0 & 0 & 0 & \cos (2 \theta) & -\sin (2 \theta) \\
0 & 0 & 0 & \sin (2 \theta) & \cos (2 \theta)
\end{array}\right] .
\end{aligned}
$$

We will denote the group of all matrices of the form in (4.9) by $G_{3}$; note that $G_{3} \cong \mathrm{SO}(2, \mathbb{R})$. Then the 3 -adapted frame fields are the smooth sections of a principal bundle $\mathcal{F}_{3} \subset \mathcal{F}_{2}$ over $M$ with fiber group $G_{3}$.

The equations (4.8) are equivalent to the condition that the Maurer-Cartan forms associated to a 3 -adapted frame field satisfy the conditions

$$
\omega_{3}^{0}=\omega_{4}^{0}=0 .
$$

Differentiating equations (4.10) yields

$$
\omega_{3}^{1} \wedge \omega_{0}^{1}+\omega_{3}^{2} \wedge \omega_{0}^{2}=\omega_{4}^{1} \wedge \omega_{0}^{1}+\omega_{4}^{2} \wedge \omega_{0}^{2}=0,
$$

and applying Cartan's lemma shows that there exist functions $h_{j k}^{i}$ on $\mathcal{F}_{3}$ such that

$$
\begin{array}{ll}
\omega_{3}^{1}=h_{31}^{1} \omega_{0}^{1}+h_{32}^{1} \omega_{0}^{2}, & \omega_{3}^{2}=h_{32}^{1} \omega_{0}^{1}+h_{32}^{2} \omega_{0}^{2}, \\
\omega_{4}^{1}=h_{41}^{1} \omega_{0}^{1}+h_{42}^{1} \omega_{0}^{2}, & \omega_{4}^{2}=h_{42}^{1} \omega_{0}^{1}+h_{42}^{2} \omega_{0}^{2} .
\end{array}
$$

Moreover, on $\mathcal{F}_{3}$, the last two relations in equations (4.7) simplify to

$$
h_{21}^{1}-h_{12}^{1}=h_{21}^{2}-h_{22}^{1}=0 .
$$

At this point, we have canonically associated to any nondegenerate, space-like centroaffine surface in $\mathbb{R}^{5} \backslash\{0\}$ a frame bundle $\mathcal{F}_{3}$ over $M$ with fiber group isomorphic to $\operatorname{SO}(2, \mathbb{R})$. Thus we have the following theorem:

Theorem 4.3. Let $\bar{f}: M \rightarrow \mathbb{R}^{5} \backslash\{0\}$ be a centroaffine immersion whose image $\Sigma=\bar{f}(M)$ is a nondegenerate, space-like centroaffine surface. Then the pullbacks of the Maurer-Cartan forms on $\operatorname{GL}(5, \mathbb{R})$ to the bundle $\mathcal{F}_{3}$ of 3-adapted frames on $\Sigma$ determine a well-defined Riemannian metric

$$
I=\left(\omega_{0}^{1}\right)^{2}+\left(\omega_{0}^{2}\right)^{2}
$$

on $\Sigma$, called the centroaffine metric. Moreover, there is a well-defined "centroaffine normal bundle" $N \Sigma$ whose fiber $N_{x} \Sigma$ at each point $x \in M$ is spanned by the vectors $\left(\mathbf{e}_{3}(x), \mathbf{e}_{4}(x)\right)$ of any 3-adapted frame at $x$, together with a well-defined Riemannian metric

$$
I_{\text {normal }}=\left(\omega_{0}^{3}\right)^{2}+\left(\omega_{0}^{4}\right)^{2}
$$

on $N \Sigma$. 
In order to obtain more information about the centroaffine metric, consider the structure equations (2.3) for the semi-basic forms $\omega_{0}^{1}, \omega_{0}^{2}$ on $M$. Based on our adaptations, it is straightforward to compute that

$$
d \omega_{0}^{1}=-\alpha \wedge \omega_{0}^{2}, \quad d \omega_{0}^{2}=\alpha \wedge \omega_{0}^{1} .
$$

Therefore, $\alpha$ is the Levi-Civita connection form associated to the centroaffine metric on $\Sigma$, and the Gauss curvature $K$ of this metric is determined by the equation

$$
d \alpha=K \omega_{0}^{1} \wedge \omega_{0}^{2}
$$

The remaining structure equations (2.3) determine relations between the functions $h_{j k}^{i}$ on $\mathcal{F}_{3}$ and their covariant derivatives with respect to $\omega_{0}^{1}, \omega_{0}^{2}$. These relations may be viewed as analogs of the Gauss and Codazzi equations for Riemannian surfaces in Euclidean space. In particular, the analog of the Gauss equation is

$$
\begin{aligned}
K= & \frac{1}{2}\left(-h_{32}^{3} h_{31}^{4}-h_{41}^{4} h_{42}^{3}+h_{41}^{3} h_{42}^{4}-h_{32}^{3} h_{42}^{4}+h_{32}^{4} h_{31}^{3}\right. \\
& \left.+h_{32}^{4} h_{42}^{3}-h_{41}^{4} h_{31}^{3}+h_{41}^{3} h_{31}^{4}+h_{31}^{1}-h_{32}^{2}+2 h_{42}^{1}\right)-\epsilon,
\end{aligned}
$$

while the remainder of the relations are partial differential equations involving the functions $h_{j k}^{i}$. An analog of Bonnet's theorem (see [6]) guarantees that, at least locally, any solution of this PDE system gives rise to a nondegenerate, space-like centroaffine surface, and that this surface

is unique up to the action of $\mathrm{GL}(5, \mathbb{R})$ on $\mathbb{R}^{5} \backslash\{0\}$. In particular, the functions $h_{j k}^{i}$ on $\mathcal{F}_{3}$ form a complete set of local invariants for such surfaces.

\section{The time-like case}

Now, suppose that the plane $P$ spanned by $\left(h^{3}, h^{4}\right)$ is time-like at every point of $M$. According to the group action (3.5), we can find a 1-adapted frame field along $\Sigma$ for which $P=P_{2}$, as in equation (3.9). Furthermore, we can then use the action by $B$ to find a 1-adapted frame field along $\Sigma$ for which

$$
h^{3}=\left[\begin{array}{ll}
1 & 0 \\
0 & 0
\end{array}\right], \quad h^{4}=\left[\begin{array}{ll}
0 & 0 \\
0 & 1
\end{array}\right] .
$$

(This choice of $h^{3}, h^{4}$ represents a null basis for $P_{2}$ with respect to the indefinite quadratic form (3.7) on $P_{2}$.)

The next step is to determine the subgroup of $G_{1}$ that preserves the conditions (5.1). To this end, first note that the plane $P_{2}$ spanned by the matrices (5.1) consists precisely of the diagonal matrices in $\operatorname{Sym}^{2}(\mathbb{R})$. A straightforward computation shows that, with $h^{3}, h^{4}$ as in (5.1), the offdiagonal components of $\operatorname{tr}\left(A^{\mathrm{T}} h^{3} A\right)$ and $\operatorname{tr}\left(A^{\mathrm{T}} h^{4} A\right)$ are equal to $a_{11} a_{12}$ and $a_{21} a_{22}$, respectively. Therefore, the action (3.5) preserves $P_{2}$ if and only if

$$
a_{11} a_{12}=a_{21} a_{22}=0 .
$$

Substituting this condition into equations (3.4) and imposing the conditions $\tilde{h}^{3}=h^{3}, \tilde{h}^{4}=h^{4}$ then shows that we must have either

$$
A=\left[\begin{array}{cc}
a_{11} & 0 \\
0 & a_{22}
\end{array}\right], \quad B=\left[\begin{array}{cc}
a_{11}^{2} & 0 \\
0 & a_{22}^{2}
\end{array}\right], \quad a_{11}, a_{22} \neq 0,
$$

or

$$
A=\left[\begin{array}{cc}
0 & a_{12} \\
a_{21} & 0
\end{array}\right], \quad B=\left[\begin{array}{cc}
0 & a_{12}^{2} \\
a_{21}^{2} & 0
\end{array}\right], \quad a_{12}, a_{21} \neq 0 .
$$


Since the latter transformation may be obtained from the former simply by interchanging $\left(\mathbf{e}_{1}, \mathbf{e}_{2}\right)$ and $\left(\mathbf{e}_{3}, \mathbf{e}_{4}\right)$, we will restrict our attention to transformations of the form (5.2), where $A, B$ are diagonal matrices and $B=A^{2}$.

Next, consider the effect of the action (3.4) on $h^{0}$. With $A=I_{2}$ and $r_{03}, r_{04}$ chosen appropriately, we can add any linear combination of $h^{3}, h^{4}$ to $h^{0}$. Thus we can find a 1-adapted frame field for which $h^{0}$ is a multiple (nonzero by the nondegeneracy assumption) of the matrix $\left[\begin{array}{ll}0 & 1 \\ 1 & 0\end{array}\right]$. Then under the action (3.4) with $A, B$ as in (5.2), we have

$$
\tilde{h}^{0}=a_{11} a_{22} h^{0}+\left[\begin{array}{cc}
-r_{03} & 0 \\
0 & -r_{04}
\end{array}\right] \text {. }
$$

Thus we can find a 1-adapted frame field along $\Sigma$ satisfying the additional condition that

$$
h^{0}=\left[\begin{array}{ll}
0 & 1 \\
1 & 0
\end{array}\right]
$$

and this condition is preserved by transformations of the form (3.4) with $A, B$ as in (5.2), such that $a_{11} a_{22}=1$ and $r_{03}=r_{04}=0$. For simplicity, we will assume that $a_{11}>0$; then we can set

$$
A=\left[\begin{array}{cc}
e^{\lambda} & 0 \\
0 & e^{-\lambda}
\end{array}\right], \quad B=\left[\begin{array}{cc}
e^{2 \lambda} & 0 \\
0 & e^{-2 \lambda}
\end{array}\right], \quad \lambda \in \mathbb{R} .
$$

Definition 5.1. Let $\Sigma=\bar{f}(M)$ be a nondegenerate, time-like centroaffine surface in $\mathbb{R}^{5} \backslash\{0\}$. A 1-adapted frame field $f: M \rightarrow \mathcal{F}_{1}$ will be called 2-adapted if it satisfies the conditions

$$
h^{3}=\left[\begin{array}{ll}
1 & 0 \\
0 & 0
\end{array}\right], \quad h^{4}=\left[\begin{array}{ll}
0 & 0 \\
0 & 1
\end{array}\right], \quad h^{0}=\left[\begin{array}{ll}
0 & 1 \\
1 & 0
\end{array}\right]
$$

at every point of $M$.

Any two 2-adapted frames $\left(\mathbf{e}_{0}, \ldots, \mathbf{e}_{4}\right),\left(\tilde{\mathbf{e}}_{0}, \ldots, \tilde{\mathbf{e}}_{4}\right)$ based at the same point $x \in M$ are related by a transformation of the form

$$
\left[\begin{array}{lllll}
\tilde{\mathbf{e}}_{0} & \tilde{\mathbf{e}}_{1} & \tilde{\mathbf{e}}_{2} & \tilde{\mathbf{e}}_{3} & \tilde{\mathbf{e}}_{4}
\end{array}\right]=\left[\begin{array}{lllll}
\mathbf{e}_{0} & \mathbf{e}_{1} & \mathbf{e}_{2} & \mathbf{e}_{3} & \mathbf{e}_{4}
\end{array}\right]\left[\begin{array}{ccccc}
1 & 0 & 0 & 0 & 0 \\
0 & e^{\lambda} & 0 & r_{13} & r_{14} \\
0 & 0 & e^{-\lambda} & r_{23} & r_{24} \\
0 & 0 & 0 & e^{2 \lambda} & 0 \\
0 & 0 & 0 & 0 & e^{-2 \lambda}
\end{array}\right] .
$$

We will denote the group of all matrices of the form in (5.4) by $G_{2}$; then the 2-adapted frame fields along $\Sigma$ are the smooth sections of a principal bundle $\mathcal{F}_{2} \subset \mathcal{F}_{1}$ over $M$ with fiber group $G_{2}$.

The equations (5.3) are equivalent to the condition that the Maurer-Cartan forms associated to a 2 -adapted frame field satisfy the conditions

$$
\omega_{1}^{3}=\omega_{0}^{1}, \quad \omega_{2}^{3}=0, \quad \omega_{1}^{4}=0, \quad \omega_{2}^{4}=\omega_{0}^{2}, \quad \omega_{1}^{0}=\omega_{0}^{2}, \quad \omega_{2}^{0}=\omega_{0}^{1} .
$$

Differentiating equations (5.5) yields

$$
\begin{array}{ll}
\left(2 \omega_{1}^{1}-\omega_{3}^{3}\right) \wedge \omega_{0}^{1}+\omega_{2}^{1} \wedge \omega_{0}^{2}=0, & \omega_{2}^{1} \wedge \omega_{0}^{1}-\omega_{4}^{3} \wedge \omega_{0}^{2}=0, \\
-\omega_{3}^{4} \wedge \omega_{0}^{1}+\omega_{1}^{2} \wedge \omega_{0}^{2}=0, & \omega_{1}^{2} \wedge \omega_{0}^{1}+\left(2 \omega_{2}^{2}-\omega_{4}^{4}\right) \wedge \omega_{0}^{2}=0, \\
\left(2 \omega_{1}^{2}-\omega_{3}^{0}\right) \wedge \omega_{0}^{1}+\left(\omega_{1}^{1}+\omega_{2}^{2}\right) \wedge \omega_{0}^{2}=0, & \left(\omega_{1}^{1}+\omega_{2}^{2}\right) \wedge \omega_{0}^{1}+\left(2 \omega_{2}^{1}-\omega_{4}^{0}\right) \wedge \omega_{0}^{2}=0 .
\end{array}
$$


Applying Cartan's lemma to these equations shows that there exists a 1-form $\alpha$ and functions $h_{j k}^{i}$ on $\mathcal{F}_{2}$ such that

$$
\begin{array}{ll}
\omega_{3}^{0}=h_{31}^{0} \omega_{0}^{1}+h_{32}^{0} \omega_{0}^{2}, & \omega_{4}^{0}=h_{41}^{0} \omega_{0}^{1}+h_{42}^{0} \omega_{0}^{2}, \\
\omega_{1}^{1}=\alpha+h_{11}^{1} \omega_{0}^{1}+h_{22}^{2} \omega_{0}^{2}, & \omega_{2}^{1}=h_{21}^{1} \omega_{0}^{1}+h_{22}^{1} \omega_{0}^{2}, \\
\omega_{1}^{2}=h_{11}^{2} \omega_{0}^{1}+h_{12}^{2} \omega_{0}^{2}, & \omega_{2}^{2}=-\alpha+h_{11}^{1} \omega_{0}^{1}+h_{22}^{2} \omega_{0}^{2}, \\
\omega_{3}^{3}=2 \alpha+h_{31}^{3} \omega_{0}^{1}+h_{32}^{3} \omega_{0}^{2}, & \omega_{4}^{3}=h_{41}^{3} \omega_{0}^{1}+h_{42}^{3} \omega_{0}^{2}, \\
\omega_{3}^{4}=h_{31}^{4} \omega_{0}^{1}+h_{32}^{4} \omega_{0}^{2}, & \omega_{4}^{4}=-2 \alpha+h_{41}^{4} \omega_{0}^{1}+h_{42}^{4} \omega_{0}^{2} .
\end{array}
$$

Moreover, the functions $h_{j k}^{i}$ satisfy the relations

$$
\begin{array}{ll}
2 h_{22}^{2}-h_{21}^{1}-h_{32}^{3}=0, & h_{22}^{1}+h_{41}^{3}=0, \\
h_{11}^{2}+h_{32}^{4}=0, & 2 h_{11}^{1}-h_{12}^{2}-h_{41}^{4}=0, \\
2 h_{11}^{1}-2 h_{12}^{2}+h_{32}^{0}=0, & 2 h_{22}^{2}-2 h_{21}^{1}+h_{41}^{0}=0 .
\end{array}
$$

If $f, \tilde{f}: M \rightarrow \mathcal{F}_{2}$ are two 2-adapted frame fields related by a transformation of the form (5.4), then we can once again use equation (2.5) to determine how the corresponding functions $h_{j k}^{i}, \tilde{h}_{j k}^{i}$ are related. As in the space-like case, the most straightforward to compute are those corresponding to the forms $\tilde{\omega}_{3}^{0}, \tilde{\omega}_{4}^{0}$. These forms appear as the coefficients of $\tilde{\mathbf{e}}_{0}=\mathbf{e}_{0}$ in the equations (2.2) for $d \tilde{\mathbf{e}}_{3}, d \tilde{\mathbf{e}}_{4}$. By applying equations (5.4) and (5.6), one can show that

$$
\left[\begin{array}{c}
\tilde{h}_{31}^{0} \\
\tilde{h}_{41}^{0}
\end{array}\right]=\left[\begin{array}{cc}
e^{2 \lambda} & 0 \\
0 & e^{-2 \lambda}
\end{array}\right]\left[\begin{array}{l}
h_{31}^{0} \\
h_{41}^{0}
\end{array}\right]+\left[\begin{array}{l}
r_{23} \\
r_{24}
\end{array}\right], \quad\left[\begin{array}{c}
\tilde{h}_{32}^{0} \\
\tilde{h}_{42}^{0}
\end{array}\right]=\left[\begin{array}{cc}
e^{2 \lambda} & 0 \\
0 & e^{-2 \lambda}
\end{array}\right]\left[\begin{array}{l}
h_{32}^{0} \\
h_{42}^{0}
\end{array}\right]+\left[\begin{array}{l}
r_{13} \\
r_{14}
\end{array}\right] .
$$

Thus we can find a 2-adapted frame field along $\Sigma$ satisfying the conditions that

$$
h_{31}^{0}=h_{32}^{0}=h_{41}^{0}=h_{42}^{0}=0,
$$

and these conditions are preserved by transformations of the form (5.4) with $r_{13}=r_{14}=r_{23}=$ $r_{24}=0$.

Definition 5.2. Let $\Sigma=\bar{f}(M)$ be a nondegenerate, time-like centroaffine surface in $\mathbb{R}^{5} \backslash\{0\}$. A 2-adapted frame field $f: M \rightarrow \mathcal{F}_{2}$ will be called 3-adapted if it satisfies the conditions

$$
h_{31}^{0}=h_{32}^{0}=h_{41}^{0}=h_{42}^{0}=0
$$

at every point of $M$.

Any two 3 -adapted frames $\left(\mathbf{e}_{0}, \ldots, \mathbf{e}_{4}\right),\left(\tilde{\mathbf{e}}_{0}, \ldots, \tilde{\mathbf{e}}_{4}\right)$ based at the same point $x \in M$ are related by a transformation of the form

$$
\left[\begin{array}{lllll}
\tilde{\mathbf{e}}_{0} & \tilde{\mathbf{e}}_{1} & \tilde{\mathbf{e}}_{2} & \tilde{\mathbf{e}}_{3} & \tilde{\mathbf{e}}_{4}
\end{array}\right]=\left[\begin{array}{lllll}
\mathbf{e}_{0} & \mathbf{e}_{1} & \mathbf{e}_{2} & \mathbf{e}_{3} & \mathbf{e}_{4}
\end{array}\right]\left[\begin{array}{ccccc}
1 & 0 & 0 & 0 & 0 \\
0 & e^{\lambda} & 0 & 0 & 0 \\
0 & 0 & e^{-\lambda} & 0 & 0 \\
0 & 0 & 0 & e^{2 \lambda} & 0 \\
0 & 0 & 0 & 0 & e^{-2 \lambda}
\end{array}\right]
$$

We will denote the group of all matrices of the form in (5.9) by $G_{3}$; note that $G_{3} \cong \mathrm{SO}^{+}(1,1)$. Then the 3 -adapted frame fields are the smooth sections of a principal bundle $\mathcal{F}_{3} \subset \mathcal{F}_{2}$ over $M$ with fiber group $G_{3}$. 
The equations (5.8) are equivalent to the condition that the Maurer-Cartan forms associated to a 3 -adapted frame field satisfy the conditions

$$
\omega_{3}^{0}=\omega_{4}^{0}=0 .
$$

Differentiating equations (5.10) yields

$$
\omega_{3}^{2} \wedge \omega_{0}^{1}+\omega_{3}^{1} \wedge \omega_{0}^{2}=\omega_{4}^{2} \wedge \omega_{0}^{1}+\omega_{4}^{1} \wedge \omega_{0}^{2}=0,
$$

and applying Cartan's lemma shows that there exist functions $h_{j k}^{i}$ on $\mathcal{F}_{3}$ such that

$$
\begin{array}{ll}
\omega_{3}^{1}=h_{31}^{1} \omega_{0}^{1}+h_{32}^{1} \omega_{0}^{2}, & \omega_{3}^{2}=h_{31}^{2} \omega_{0}^{1}+h_{31}^{1} \omega_{0}^{2}, \\
\omega_{4}^{1}=h_{41}^{1} \omega_{0}^{1}+h_{42}^{1} \omega_{0}^{2}, & \omega_{4}^{2}=h_{41}^{2} \omega_{0}^{1}+h_{41}^{1} \omega_{0}^{2} .
\end{array}
$$

Moreover, on $\mathcal{F}_{3}$, the last two relations in equations (5.7) simplify to

$$
h_{11}^{1}-h_{12}^{2}=h_{22}^{2}-h_{21}^{1}=0 .
$$

At this point, we have canonically associated to any nondegenerate, time-like centroaffine surface in $\mathbb{R}^{5} \backslash\{0\}$ a frame bundle $\mathcal{F}_{3}$ over $M$ with fiber group isomorphic to $\mathrm{SO}^{+}(1,1)$. Thus we have the following theorem:

Theorem 5.3. Let $\bar{f}: M \rightarrow \mathbb{R}^{5} \backslash\{0\}$ be a centroaffine immersion whose image $\Sigma=\bar{f}(M)$ is a nondegenerate, time-like centroaffine surface. Then the pullbacks of the Maurer-Cartan forms on $\mathrm{GL}(5, \mathbb{R})$ to the bundle $\mathcal{F}_{3}$ of 3-adapted frames on $\Sigma$ determine a well-defined Lorentzian metric

$$
I=2 \omega_{0}^{1} \omega_{0}^{2}
$$

on $\Sigma$, called the centroaffine metric. Moreoever, there is a well-defined "centroaffine normal bundle" $N \Sigma$ whose fiber $N_{x} \Sigma$ at each point $x \in M$ is spanned by the vectors $\left(\mathbf{e}_{3}(x), \mathbf{e}_{4}(x)\right)$ of any 3-adapted frame at $x$, together with a well-defined Lorentzian metric

$$
I_{\text {normal }}=2 \omega_{0}^{3} \omega_{0}^{4}
$$

on $N \Sigma$.

In order to obtain more information about the centroaffine metric, consider the structure equations (2.3) for the semi-basic forms $\omega_{0}^{1}, \omega_{0}^{2}$ on $M$. Based on our adaptations, it is straightforward to compute that

$$
d \omega_{0}^{1}=-\alpha \wedge \omega_{0}^{1}, \quad d \omega_{0}^{2}=\alpha \wedge \omega_{0}^{2} .
$$

Therefore, $\alpha$ is the Levi-Civita connection form associated to the centroaffine metric on $\Sigma$, and the Gauss curvature $K$ of this metric is determined by the equation

$$
d \alpha=K \omega_{0}^{1} \wedge \omega_{0}^{2}
$$

As in the space-like case, the remaining structure equations (2.3) determine relations between the functions $h_{j k}^{i}$ on $\mathcal{F}_{3}$ and their covariant derivatives with respect to $\omega_{0}^{1}, \omega_{0}^{2}$. The analog of the Gauss equation is

$$
K=h_{41}^{3} h_{32}^{4}-h_{32}^{3} h_{41}^{4}+\frac{1}{2}\left(h_{32}^{1}+h_{41}^{2}\right)-1,
$$

while the remainder of the relations are partial differential equations involving the functions $h_{j k}^{i}$. As in the space-like case, any solution of this PDE system locally gives rise to a nondegenerate, time-like centroaffine surface; this surface is unique up to the action of $\mathrm{GL}(5, \mathbb{R})$ on $\mathbb{R}^{5} \backslash\{0\}$, and the functions $h_{j k}^{i}$ on $\mathcal{F}_{3}$ form a complete set of local invariants for such surfaces. 


\section{Homogeneous examples}

The goal of this section is to give a complete classification (up to the GL(5, $\mathbb{R}$ )-action on $\mathbb{R}^{5} \backslash\{0\}$ ) of the homogeneous examples of space-like and time-like nondegenerate centroaffine surfaces in $\mathbb{R}^{5} \backslash\{0\}$. First we must define precisely what we mean by the term "homogeneous". Because any such surface $\Sigma=\bar{f}(M)$ has a well-defined Riemannian or Lorentzian metric, any symmetry of $\Sigma$ must preserve the centroaffine metric on $\Sigma$ and hence must in fact be an isometry of $\Sigma$ with its centroaffine metric. Furthermore, if the group of symmetries of $\Sigma$ acts transitively, then the centroaffine metric must have constant Gauss curvature $K$. As is well-known, the maximal isometry group of any Riemannian or Lorentzian surface has dimension less than or equal to three, and in the maximal case the isometry group acts transitively on the orthonormal frame bundle. Thus we will use the following definition:

Definition 6.1. Let $\mathcal{F}_{3}$ be the bundle of 3 -adapted frames along a nondegenerate, space-like or time-like centroaffine surface $\Sigma=\bar{f}(M)$ in $\mathbb{R}^{5} \backslash\{0\}$. A diffeomorphism $\phi: \mathcal{F}_{3} \rightarrow \mathcal{F}_{3}$ is called a symmetry of $\Sigma$ if $\phi^{*} \Omega=\Omega$; i.e., if $\phi$ preserves the Maurer-Cartan forms on $\mathcal{F}_{3}$. A nondegenerate space-like or time-like centroaffine surface $\Sigma=\bar{f}(M)$ will be called homogeneous if the group of symmetries of $\Sigma$ is a 3 -dimensional Lie group that acts transitively on $\mathcal{F}_{3}$.

Remark 6.2. It might also be of interest to consider the slightly less restrictive assumption that $\Sigma$ has a 2-dimensional group of symmetries that acts transitively on the base manifold $M$, but we will not consider this scenario here.

Our procedure for classifying the homogeneous examples is as follows. Observe that if $\Sigma$ is homogeneous, then all the structure functions $h_{j k}^{i}$ must be constant on the bundle $\mathcal{F}_{3}$ of 3 -adapted frames on $\Sigma$. When this condition is imposed, the structure equations (2.3) become algebraic relations among the constants $h_{j k}^{i}$. Given any solution to these relations, the structure equations (2.3) imply that the corresponding Maurer-Cartan form $\Omega=\left[\omega_{j}^{i}\right]$ takes values in a 3 -dimensional Lie algebra $\mathfrak{g}$ which is realized explicitly as a Lie subalgebra of $\mathfrak{g l}(5, \mathbb{R})$. Thus $\Omega$ is also the Maurer-Cartan form of the connected Lie group $G \subset \mathrm{GL}(5, \mathbb{R})$ generated by exponentiating $\mathfrak{g}$, and this equivalence of the Maurer-Cartan forms on $\mathcal{F}_{3}$ with those on $G$ implies that $\mathcal{F}_{3}$ is a homogeneous space for $G$; indeed, $G$ must be precisely the symmetry group that was assumed to act transitively on $\mathcal{F}_{3}$.

Now, choose any point $\mathbf{f}_{0}=\left(\mathbf{e}_{0}, \mathbf{e}_{1}, \mathbf{e}_{2}, \mathbf{e}_{3}, \mathbf{e}_{4}\right) \in \mathcal{F}_{3}$. Recall that we can view $\mathbf{f}_{0}$ as an element of $\operatorname{GL}(5, \mathbb{R})$. The centroaffine surface $\Sigma$ is equivalent via the $\operatorname{GL}(5, \mathbb{R})$-action to the surface $\widetilde{\Sigma}=\mathbf{f}_{0}^{-1} \cdot \Sigma$, and the bundle $\widetilde{\mathcal{F}}_{3}$ of 3 -adapted frames over $\widetilde{\Sigma}$ is given by $\widetilde{\mathcal{F}}_{3}=\mathbf{f}_{0}^{-1} \cdot \mathcal{F}_{3}$. So without loss of generality, we may assume that $\mathbf{f}_{0}$ is the identity matrix $I_{5}$. With this assumption, the tangent space $T_{\mathbf{f}_{0}} \mathcal{F}_{3}$ is equal to the Lie algebra $\mathfrak{g}$, and $\mathcal{F}_{3}$ must in fact be equal to $G$. Finally, $\Sigma$ is given by the image of $G$ under the projection (2.1).

In order to carry out this procedure, we consider the space-like and time-like cases separately.

\subsection{Space-like homogeneous examples}

From the adaptations of Section 4 , the matrix $\Omega=\left[\omega_{j}^{i}\right]$ of Maurer-Cartan forms on $\mathcal{F}_{3}$ may be written as

$$
\Omega=\left[\begin{array}{ccccc}
0 & 0 & 0 & 0 & 0 \\
0 & 0 & 1 & 0 & 0 \\
0 & -1 & 0 & 0 & 0 \\
0 & 0 & 0 & 0 & 2 \\
0 & 0 & 0 & -2 & 0
\end{array}\right] \alpha+\left[\begin{array}{ccccc}
0 & \epsilon & 0 & 0 & 0 \\
1 & \frac{1}{2}\left(h_{31}^{3}+h_{42}^{3}\right)-h_{32}^{4}+h_{41}^{4} & \frac{1}{2}\left(h_{32}^{3}-h_{41}^{3}\right) & h_{31}^{1} & h_{41}^{1} \\
0 & \frac{1}{2}\left(h_{32}^{3}-h_{41}^{3}\right) & \frac{1}{2}\left(h_{31}^{3}+h_{42}^{3}\right) & h_{32}^{1} & h_{42}^{1} \\
0 & 1 & 0 & h_{31}^{3} & h_{41}^{3} \\
0 & 0 & 1 & h_{31}^{4} & h_{41}^{4}
\end{array}\right] \omega_{0}^{1}
$$




$$
+\left[\begin{array}{ccccc}
0 & 0 & \epsilon & 0 & 0 \\
0 & \frac{1}{2}\left(h_{32}^{3}-h_{41}^{3}\right) & \frac{1}{2}\left(h_{31}^{3}+h_{42}^{3}\right) & h_{32}^{1} & h_{42}^{1} \\
1 & \frac{1}{2}\left(h_{31}^{3}+h_{42}^{3}\right) & \frac{1}{2}\left(h_{32}^{3}-h_{41}^{3}\right)+h_{31}^{4}+h_{42}^{4} & h_{32}^{2} & h_{42}^{2} \\
0 & 0 & -1 & h_{32}^{3} & h_{42}^{3} \\
0 & 1 & 0 & h_{32}^{4} & h_{42}^{4}
\end{array}\right] \omega_{0}^{2}
$$

while the structure equations (2.3) may be written as

$$
d \Omega=-\Omega \wedge \Omega .
$$

Substituting (6.1) into equation (6.2) and imposing the condition that all the functions $h_{j k}^{i}$ are constant leads to a system of 25 algebraic equations (some linear, some quadratic) for the 14 unknown constants $h_{j k}^{i}$. A somewhat tedious, but straightforward, computation shows that this system has precisely two solutions, one for $\epsilon=1$ and one for $\epsilon=-1$. These are described in the following two examples.

Example 6.3. When $\epsilon=1$, the unique solution to equation (6.2) with all $h_{j k}^{i}$ constant is

$$
\Omega=\left[\begin{array}{ccccc}
0 & 0 & 0 & 0 & 0 \\
0 & 0 & 1 & 0 & 0 \\
0 & -1 & 0 & 0 & 0 \\
0 & 0 & 0 & 0 & 2 \\
0 & 0 & 0 & -2 & 0
\end{array}\right] \alpha+\left[\begin{array}{ccccc}
0 & 1 & 0 & 0 & 0 \\
1 & 0 & 0 & \frac{1}{3} & 0 \\
0 & 0 & 0 & 0 & \frac{1}{3} \\
0 & 1 & 0 & 0 & 0 \\
0 & 0 & 1 & 0 & 0
\end{array}\right] \omega_{0}^{1}+\left[\begin{array}{ccccc}
0 & 0 & 1 & 0 & 0 \\
0 & 0 & 0 & 0 & \frac{1}{3} \\
1 & 0 & 0 & -\frac{1}{3} & 0 \\
0 & 0 & -1 & 0 & 0 \\
0 & 1 & 0 & 0 & 0
\end{array}\right] \omega_{0}^{2} .
$$

Furthermore, the Gauss equation (4.11) implies that the centroaffine metric has Gauss curvature $K=-\frac{1}{3}$.

Denote the matrices in equation (6.3) by $M_{0}, M_{1}, M_{2}$, respectively, so that

$$
\Omega=M_{0} \alpha+M_{1} \omega_{0}^{1}+M_{2} \omega_{0}^{2}
$$

It is straightforward to compute that

$$
\left[M_{0}, M_{1}\right]=-M_{2}, \quad\left[M_{1}, M_{2}\right]=\frac{1}{3} M_{0}, \quad\left[M_{2}, M_{0}\right]=-M_{1} .
$$

These bracket relations imply that the Lie algebra $\mathfrak{g} \subset \mathfrak{g l}(5, \mathbb{R})$ spanned by $\left(M_{0}, M_{1}, M_{2}\right)$ is isomorphic to $\mathfrak{s o}(1,2)$. (They also suggest that a more natural basis might be obtained by multiplying each of $M_{1}, M_{2}$ by $\sqrt{3}$.) Furthermore, it is straightforward to check that $\mathfrak{g}$ acts irreducibly on $\mathbb{R}^{5} \backslash\{0\}$. It is well-known (see, e.g., [2, p. 149]) that $\mathfrak{s o}(1,2)$ has a unique irreducible 5-dimensional representation and that this representation arises from a (unique) irreducible representation of $\mathrm{SO}^{+}(1,2)$; it follows that the Lie group $G \subset \mathrm{GL}(5, \mathbb{R})$ corresponding to the Lie algebra $\mathfrak{g}$ is isomorphic to $\mathrm{SO}^{+}(1,2)$.

The easiest way to compute a local parametrization for $G$ - and hence for $\Sigma$ - is to compute the 1-parameter subgroups generated by $M_{0}, M_{1}, M_{2}$ and take products of the resulting group elements.

Warning. This must be done carefully in order to ensure that the resulting products cover the entire group $G$, which in turn guarantees that the resulting parametrization is surjective onto $\Sigma$. The subtlety of this issue can already be seen in the standard representation for $\mathfrak{s o}(1,2)$ : the basis

$$
\bar{M}_{0}=\left[\begin{array}{ccc}
0 & 0 & 0 \\
0 & 0 & 1 \\
0 & -1 & 0
\end{array}\right], \quad \bar{M}_{1}=\left[\begin{array}{ccc}
0 & 1 & 0 \\
1 & 0 & 0 \\
0 & 0 & 0
\end{array}\right], \quad \bar{M}_{2}=\left[\begin{array}{ccc}
0 & 0 & 1 \\
0 & 0 & 0 \\
1 & 0 & 0
\end{array}\right]
$$


has the same bracket relations as $\left(M_{0}, \sqrt{3} M_{1}, \sqrt{3} M_{2}\right)$, and exponentiating this basis yields the 1-parameter subgroups

$$
\begin{aligned}
& \bar{g}_{0}(t)=\left[\begin{array}{ccc}
1 & 0 & 0 \\
0 & \cos (t) & \sin (t) \\
0 & -\sin (t) & \cos (t)
\end{array}\right] \\
& \bar{g}_{1}(u)=\left[\begin{array}{ccc}
\cosh (u) & \sinh (u) & 0 \\
\sinh (u) & \cosh (u) & 0 \\
0 & 0 & 1
\end{array}\right], \quad \bar{g}_{2}(v)=\left[\begin{array}{ccc}
\cosh (v) & 0 & \sinh (v) \\
0 & 1 & 0 \\
\sinh (v) & 0 & \cosh (v)
\end{array}\right] \text {. }
\end{aligned}
$$

Now consider the following two maps $f_{1}, f_{2}: \mathbb{R}^{3} \rightarrow \mathrm{SO}^{+}(1,2)$, which are obtained by multiplying the elements $\bar{g}_{0}(t), \bar{g}_{1}(u), \bar{g}_{2}(v)$ in different orders:

$$
\begin{aligned}
& f_{1}(u, v, t)=\bar{g}_{1}(u) \bar{g}_{2}(v) \bar{g}_{0}(t) \\
& =\left[\begin{array}{ccc}
\cosh (u) \cosh (v) & \sinh (u) \cos (t) & \sinh (u) \sin (t) \\
& -\cosh (u) \sinh (v) \sin (t) & +\cosh (u) \sinh (v) \cos (t) \\
\sinh (u) \cosh (v) & -\sinh (u) \sinh (v) \sin (t) & +\sinh (u) \sinh (v) \cos (t) \\
\sinh (v) & -\cosh (v) \sin (t) & \cosh (v) \cos (t)
\end{array}\right], \\
& f_{2}(u, v, t)=\bar{g}_{1}(u) \bar{g}_{0}(t) \bar{g}_{2}(v) \\
& =\left[\begin{array}{ccc}
\cosh (u) \cosh (v) & \sinh (u) \cos (t) & +\sinh (u) \cosh (v) \sin (t) \\
+\sinh (u) \sinh (v) \sin (t) & \sinh (u) \sinh (v) \\
\sinh (u) \cosh (v) & & \cosh (u) \cos (t) \\
+\cosh (u) \sinh (v) \sin (t) & +\cosh (u) \cosh (v) \sin (t) \\
\sinh (v) \cos (t) & -\sin (t) & \cosh (v) \cos (t)
\end{array}\right] .
\end{aligned}
$$

It is not difficult to show that $f_{1}$ is surjective onto $\mathrm{SO}^{+}(1,2)$, whereas we can see from the middle column that $f_{2}$ is not. Thus we must perform this construction with care.

Now, the obvious correspondence

$$
\bar{M}_{0} \leftrightarrow M_{0}, \quad \bar{M}_{1} \leftrightarrow \sqrt{3} M_{1}, \quad \bar{M}_{2} \leftrightarrow \sqrt{3} M_{2},
$$

defines a Lie algebra isomorphism between the standard representation of $\mathfrak{s o}(1,2)$ and our Lie algebra $\mathfrak{g} \subset \mathfrak{g l}(5, \mathbb{R})$. Therefore, the surjectivity of the map $f_{1}$ above implies that the analogous map $f: \mathbb{R}^{3} \rightarrow G$ will also be surjective onto $G$, and it follows that the map $\bar{f}=\pi \circ f$ will be surjective onto $\Sigma$. The map $\bar{f}$ will also turn out to be independent of $t$, and when regarded as a function of the two variables $(u, v)$ it will define a surjective parametrization $\bar{f}: \mathbb{R}^{2} \rightarrow \Sigma$.

With these considerations in mind, define the 1-parameter subgroups

$$
\begin{aligned}
g_{0}(t) & =\exp \left(t M_{0}\right)=\left[\begin{array}{ccccc}
1 & 0 & 0 & 0 & 0 \\
0 & \cos (t) & \sin (t) & 0 & 0 \\
0 & -\sin (t) & \cos (t) & 0 & 0 \\
0 & 0 & 0 & \cos (2 t) & \sin (2 t) \\
0 & 0 & 0 & -\sin (2 t) & \cos (2 t)
\end{array}\right], \\
g_{1}(u) & =\exp \left(u \sqrt{3} M_{1}\right) \\
= & {\left[\begin{array}{ccccc}
\frac{1}{4}(3 \cosh (2 u)+1) & \frac{\sqrt{3}}{2} \sinh (2 u) & 0 & \frac{1}{4}(\cosh (2 u)-1) & 0 \\
\frac{\sqrt{3}}{2} \sinh (2 u) & \cosh (2 u) & 0 & \frac{1}{2 \sqrt{3}} \sinh (2 u) & 0 \\
0 & 0 & \cosh (u) & 0 & \frac{1}{\sqrt{3}} \sinh (u) \\
\frac{3}{4}(\cosh (2 u)-1) & \frac{\sqrt{3}}{2} \sinh (2 u) & 0 & \frac{1}{4}(\cosh (2 u)+3) & 0 \\
0 & 0 & \sqrt{3} \sinh (u) & 0 & \cosh (u)
\end{array}\right], }
\end{aligned}
$$




$$
\begin{aligned}
g_{2}(v) & =\exp \left(v \sqrt{3} M_{2}\right) \\
& =\left[\begin{array}{ccccc}
\frac{1}{4}(3 \cosh (2 v)+1) & 0 & \frac{\sqrt{3}}{2} \sinh (2 v) & \frac{1}{4}(1-\cosh (2 v)) & 0 \\
0 & \cosh (v) & 0 & 0 & \frac{1}{\sqrt{3}} \sinh (v) \\
\frac{\sqrt{3}}{2} \sinh (2 v) & 0 & \cosh (2 v) & -\frac{1}{2 \sqrt{3}} \sinh (2 v) & 0 \\
\frac{3}{4}(1-\cosh (2 v)) & 0 & -\frac{\sqrt{3}}{2} \sinh (2 v) & \frac{1}{4}(\cosh (2 v)+3) & 0 \\
0 & \sqrt{3} \sinh (v) & 0 & 0 & \cosh (v)
\end{array}\right] .
\end{aligned}
$$

Then set

$$
\bar{f}(u, v, t)=\pi\left(g_{1}(u) \cdot g_{2}(v) \cdot g_{0}(t)\right)=\left[\begin{array}{c}
\frac{1}{2}\left(3 \cosh ^{2}(u) \cosh ^{2}(v)-1\right) \\
\sqrt{3} \sinh (u) \cosh (u) \cosh ^{2}(v) \\
\sqrt{3} \cosh (u) \sinh (v) \cosh (v) \\
\frac{3}{2}\left(\cosh ^{2}(v)\left(\cosh ^{2}(u)-2\right)+1\right) \\
3 \sinh (u) \sinh (v) \cosh (v)
\end{array}\right] .
$$

It follows from the discussion above that $\bar{f}$ is a surjective map onto $\Sigma$. Moreover, it is straightforward to check that the tangent vectors $\bar{f}_{u}, \bar{f}_{v}$ are linearly independent for all $(u, v) \in \mathbb{R}^{2}$; therefore $\bar{f}$ parametrizes a smooth surface $\Sigma \subset \mathbb{R}^{5} \backslash\{0\}$, as expected.

We can compute the centroaffine metric on $\Sigma$ explicitly as follows. Let $f: \mathbb{R}^{3} \rightarrow G$ be the map corresponding to (6.4); i.e.,

$$
f(u, v, t)=g_{1}(u) \cdot g_{2}(v) \cdot g_{0}(t) .
$$

Then we have

$$
\Omega=M_{0} \alpha+M_{1} \omega_{0}^{1}+M_{2} \omega_{0}^{2}=f^{-1} d f
$$

Comparing these two expressions for $\Omega$ shows that

$$
\omega_{0}^{1}=\sqrt{3}(\cosh (v) \cos (t) d u-\sin (t) d v), \quad \omega_{0}^{2}=\sqrt{3}(\cosh (v) \sin (t) d u+\cos (t) d v) .
$$

Therefore, the centroaffine metric on $\Sigma$ is given by

$$
I=\left(\omega_{0}^{1}\right)^{2}+\left(\omega_{0}^{2}\right)^{2}=3\left(\cosh ^{2}(v) d u^{2}+d v^{2}\right) .
$$

As expected, this metric has constant Gauss curvature $K=-\frac{1}{3}$. Topologically, $\Sigma$ is simply connected and isometric to the hyperbolic plane $\mathbb{H}$ of "radius" $\sqrt{3}$, while $\mathcal{F}_{3}$ is isomorphic to the orthonormal frame bundle of $\mathbb{H}$, with $\pi$ as the projection map.

In fact, we can describe $\Sigma$ more intrinsically: if we denote the coordinates of a point $\mathbf{x} \in$ $\mathbb{R}^{5} \backslash\{0\}$ as $\left(x_{0}, \ldots, x_{4}\right)$, then the coordinates of $\bar{f}(u, v)$ satisfy the quadratic equations

$$
\begin{aligned}
& x_{1}\left(x_{0}-x_{3}-1\right)-x_{2} x_{4}=0, \quad x_{2}\left(x_{0}+x_{3}-1\right)-x_{1} x_{4}=0, \\
& \left(4 x_{0}-1\right)^{2}-12 x_{1}^{2}-12 x_{2}^{2}-9=0 .
\end{aligned}
$$

Therefore, $\Sigma$ is contained in the (real) algebraic variety $X \subset \mathbb{R}^{5} \backslash\{0\}$ defined by equations (6.5). $\Sigma$ is not, however, equal to all of $X$; for instance, $X$ contains an affine plane consisting of all points of the form $\left(1,0,0, x_{3}, x_{4}\right)$, and this plane intersects $\Sigma$ only when $x_{3}=x_{4}=0$. Projections of $\Sigma$ to the $\left(x_{1}, x_{2}, x_{0}\right),\left(x_{1}, x_{2}, x_{3}\right)$, and $\left(x_{1}, x_{2}, x_{4}\right)$ coordinate 3 -planes are shown in Fig. 1. 

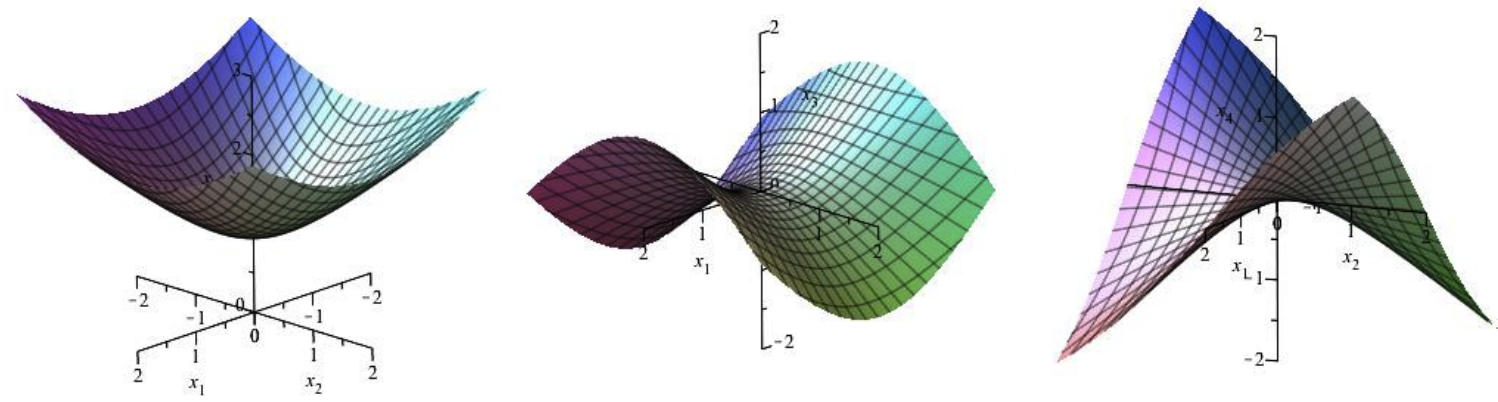

Figure 1. Projections of surface from Example 6.3 to 3-D subspaces.

Example 6.4. When $\epsilon=-1$, the unique solution to equation (6.2) with all $h_{j k}^{i}$ constant is

$$
\Omega=\left[\begin{array}{ccccc}
0 & 0 & 0 & 0 & 0 \\
0 & 0 & 1 & 0 & 0 \\
0 & -1 & 0 & 0 & 0 \\
0 & 0 & 0 & 0 & 2 \\
0 & 0 & 0 & -2 & 0
\end{array}\right] \alpha+\left[\begin{array}{ccccc}
0 & -1 & 0 & 0 & 0 \\
1 & 0 & 0 & -\frac{1}{3} & 0 \\
0 & 0 & 0 & 0 & -\frac{1}{3} \\
0 & 1 & 0 & 0 & 0 \\
0 & 0 & 1 & 0 & 0
\end{array}\right] \omega_{0}^{1}+\left[\begin{array}{ccccc}
0 & 0 & -1 & 0 & 0 \\
0 & 0 & 0 & 0 & -\frac{1}{3} \\
1 & 0 & 0 & \frac{1}{3} & 0 \\
0 & 0 & -1 & 0 & 0 \\
0 & 1 & 0 & 0 & 0
\end{array}\right] \omega_{0}^{2}
$$

Furthermore, the Gauss equation (4.11) implies that the centroaffine metric has Gauss curvature $K=\frac{1}{3}$.

As in the previous example, denote the matrices in equation (6.6) by $M_{0}, M_{1}, M_{2}$, respectively. Then we have

$$
\left[M_{0}, M_{1}\right]=-M_{2}, \quad\left[M_{1}, M_{2}\right]=-\frac{1}{3} M_{0}, \quad\left[M_{2}, M_{0}\right]=-M_{1} .
$$

These bracket relations imply that the Lie algebra $\mathfrak{g} \subset \mathfrak{g l}(5, \mathbb{R})$ spanned by $\left(M_{0}, M_{1}, M_{2}\right)$ is isomorphic to $\mathfrak{s o}(3, \mathbb{R})$. Furthermore, it is straightforward to check that $\mathfrak{g}$ acts irreducibly on $\mathbb{R}^{5} \backslash$ $\{0\}$. Similarly to the previous example, $\mathfrak{s o}(3, \mathbb{R})$ has a unique irreducible 5-dimensional representation, and this representation arises from a (unique) irreducible representation of $\mathrm{SO}(3, \mathbb{R})$; it follows that the Lie group $G \subset \mathrm{GL}(5, \mathbb{R})$ corresponding to the Lie algebra $\mathfrak{g}$ is isomorphic to $\mathrm{SO}(3, \mathbb{R})$.

We will compute a local parametrization for $\Sigma$ as in the previous example: compute the 1-parameter subgroups of $G$ generated by $M_{0}, M_{1}, M_{2}$ and take products of the resulting group elements. Ensuring the surjectivity of the resulting parametrization is easier than in the previous example. First, observe that a basis $\left(\bar{M}_{0}, \bar{M}_{1}, \bar{M}_{2}\right)$ for the standard representation of $\mathfrak{s o}(3, \mathbb{R})$ with the same bracket relations as $\left(M_{0}, \sqrt{3} M_{1}, \sqrt{3} M_{2}\right)$ is given by

$$
\bar{M}_{0}=\left[\begin{array}{ccc}
0 & 0 & 0 \\
0 & 0 & 1 \\
0 & -1 & 0
\end{array}\right], \quad \bar{M}_{1}=\left[\begin{array}{ccc}
0 & -1 & 0 \\
1 & 0 & 0 \\
0 & 0 & 0
\end{array}\right], \quad \bar{M}_{2}=\left[\begin{array}{ccc}
0 & 0 & -1 \\
0 & 0 & 0 \\
1 & 0 & 0
\end{array}\right] .
$$

Exponentiating this basis yields the 1-parameter subgroups

$$
\begin{array}{rlr}
\bar{g}_{0}(t) & =\left[\begin{array}{ccc}
1 & 0 & 0 \\
0 & \cos (t) & \sin (t) \\
0 & -\sin (t) & \cos (t)
\end{array}\right], & \\
\bar{g}_{1}(u) & =\left[\begin{array}{ccc}
\cos (u) & -\sin (u) & 0 \\
\sin (u) & \cos (u) & 0 \\
0 & 0 & 1
\end{array}\right], & \bar{g}_{2}(v)=\left[\begin{array}{ccc}
\cos (v) & 0 & -\sin (v) \\
0 & 1 & 0 \\
\sin (v) & 0 & \cos (v)
\end{array}\right] .
\end{array}
$$


Then the map $f: \mathbb{R}^{3} \rightarrow \mathrm{SO}(3, \mathbb{R})$ defined by

$$
\begin{aligned}
f(u, v, t) & =\bar{g}_{1}(u) \bar{g}_{2}(v) \bar{g}_{0}(t) \\
& =\left[\begin{array}{ccc}
\cos (u) \cos (v) & -\sin (u) \cos (t) & -\sin (u) \sin (t) \\
& +\cos (u) \sin (v) \sin (t) & -\cos (u) \sin (v) \cos (t) \\
\sin (u) \cos (v) & +\cos (u) \cos (t) & \cos (u) \sin (t) \\
\sin (v) & -\cos (v) \sin (t) & \cos (v) \cos (t)
\end{array}\right]
\end{aligned}
$$

is easily seen to be surjective onto $\mathrm{SO}(3, \mathbb{R})$. Thus the analogous map $f: \mathbb{R}^{3} \rightarrow G$ will be surjective onto $G$, and the map $\bar{f}=\pi \circ f$ will be surjective onto $\Sigma$.

So, define the 1-parameter subgroups

$$
\begin{aligned}
& g_{0}(t)=\exp \left(t M_{0}\right)=\left[\begin{array}{ccccc}
1 & 0 & 0 & 0 & 0 \\
0 & \cos (t) & \sin (t) & 0 & 0 \\
0 & -\sin (t) & \cos (t) & 0 & 0 \\
0 & 0 & 0 & \cos (2 t) & \sin (2 t) \\
0 & 0 & 0 & -\sin (2 t) & \cos (2 t)
\end{array}\right], \\
& g_{1}(u)=\exp \left(u \sqrt{3} M_{1}\right) \\
& =\left[\begin{array}{ccccc}
\frac{1}{4}(3 \cos (2 u)+1) & -\frac{\sqrt{3}}{2} \sin (2 u) & 0 & \frac{1}{4}(1-\cos (2 u)) & 0 \\
\frac{\sqrt{3}}{2} \sin (2 u) & \cos (2 u) & 0 & -\frac{1}{2 \sqrt{3}} \sin (2 u) & 0 \\
0 & 0 & \cos (u) & 0 & -\frac{1}{\sqrt{3}} \sin (u) \\
\frac{3}{4}(1-\cos (2 u)) & \frac{\sqrt{3}}{2} \sin (2 u) & 0 & \frac{1}{4}(\cos (2 u)+3) & 0 \\
0 & 0 & \sqrt{3} \sin (u) & 0 & \cos (u)
\end{array}\right], \\
& g_{2}(v)=\exp \left(v \sqrt{3} M_{2}\right) \\
& =\left[\begin{array}{ccccc}
\frac{1}{4}(3 \cos (2 v)+1) & 0 & -\frac{\sqrt{3}}{2} \sin (2 v) & \frac{1}{4}(\cos (2 v)-1) & 0 \\
0 & \cos (v) & 0 & 0 & -\frac{1}{\sqrt{3}} \sin (v) \\
\frac{\sqrt{3}}{2} \sin (2 v) & 0 & \cos (2 v) & \frac{1}{2 \sqrt{3}} \sin (2 v) & 0 \\
\frac{3}{4}(\cos (2 v)-1) & 0 & -\frac{\sqrt{3}}{2} \sin (2 v) & \frac{1}{4}(\cos (2 v)+3) & 0 \\
0 & \sqrt{3} \sin (v) & 0 & 0 & \cos (v)
\end{array}\right] .
\end{aligned}
$$

Then set

$$
\bar{f}(u, v, t)=\pi\left(g_{1}(u) \cdot g_{2}(v) \cdot g_{0}(t)\right)=\left[\begin{array}{c}
\frac{1}{2}\left(3 \cos ^{2}(u) \cos ^{2}(v)-1\right) \\
\sqrt{3} \sin (u) \cos (u) \cos ^{2}(v) \\
\sqrt{3} \cos (u) \sin (v) \cos (v) \\
\frac{3}{2}\left(\cos ^{2}(v)\left(2-\cos ^{2}(u)\right)-1\right) \\
3 \sin (u) \sin (v) \cos (v)
\end{array}\right] .
$$

It follows from the discussion above that $\bar{f}$ is a surjective map onto $\Sigma$, and we see that $\bar{f}$ is also independent of $t$. Unlike in the previous example, the tangent vectors $\bar{f}_{u}, \bar{f}_{v}$ are not linearly independent for all $(u, v) \in \mathbb{R}^{2}$; indeed, $\bar{f}_{u}=0$ whenever $v$ is an odd multiple of $\frac{\pi}{2}$. Nevertheless, the restriction of $\bar{f}$ to some neighborhood of the point $(u, v)=(0,0)$ is a smooth embedding, and then homogeneity implies that $\Sigma$ is smooth everywhere.

We can compute the centroaffine metric on $\Sigma$ as in the previous example. Let $f: \mathbb{R}^{3} \rightarrow G$ be the map corresponding to (6.7); i.e.,

$$
f(u, v, t)=g_{1}(u) \cdot g_{2}(v) \cdot g_{0}(t) .
$$


Then we have

$$
\Omega=M_{0} \alpha+M_{1} \omega_{0}^{1}+M_{2} \omega_{0}^{2}=f^{-1} d f
$$

Comparing these two expressions for $\Omega$ shows that, whenever $\cos (v) \neq 0$,

$$
\omega_{0}^{1}=\sqrt{3}(\cos (v) \cos (t) d u-\sin (t) d v), \quad \omega_{0}^{2}=\sqrt{3}(\cos (v) \sin (t) d u+\cos (t) d v) .
$$

Therefore, the centroaffine metric on $\Sigma$ is given by

$$
I=\left(\omega_{0}^{1}\right)^{2}+\left(\omega_{0}^{2}\right)^{2}=3\left(\cos ^{2}(v) d u^{2}+d v^{2}\right) .
$$

As expected, this metric has constant Gauss curvature $K=\frac{1}{3}$. Topologically, $\Sigma$ is simply connected and isometric to the sphere $S$ of radius $\sqrt{3}$, while $\mathcal{F}_{3}$ is isomorphic to the orthonormal frame bundle of $S$, with $\pi$ as the projection map.

As in the previous example, we can show that $\Sigma$ is contained in the intersection of three quadric hypersurfaces in $\mathbb{R}^{5} \backslash\{0\}$. The coordinates of $\bar{f}(u, v)$ satisfy the quadratic equations

$$
\begin{aligned}
& x_{1}\left(x_{0}+x_{3}-1\right)+x_{2} x_{4}=0, \quad x_{2}\left(x_{0}-x_{3}-1\right)+x_{1} x_{4}=0, \\
& \left(4 x_{0}-1\right)^{2}+12 x_{1}^{2}+12 x_{2}^{2}-9=0 .
\end{aligned}
$$

Therefore, $\Sigma$ is contained in the (real) algebraic variety $X \subset \mathbb{R}^{5} \backslash\{0\}$ defined by equations (6.8). Once again, $\Sigma$ is not equal to all of $X$ : the variety $X$ contains affine planes consisting of all points of the form $\left(1,0,0, x_{3}, x_{4}\right)$ or $\left(-\frac{1}{2}, 0,0, x_{3}, x_{4}\right)$; the former intersects $\Sigma$ only when $x_{3}=x_{4}=0$, and the latter intersects $\Sigma$ only when $x_{3}^{2}+x_{4}^{2}=\frac{9}{4}$. Projections of $\Sigma$ to the $\left(x_{1}, x_{2}, x_{0}\right),\left(x_{1}, x_{2}, x_{3}\right)$, and $\left(x_{1}, x_{2}, x_{4}\right)$ coordinate 3 -planes are shown in Fig. 2.
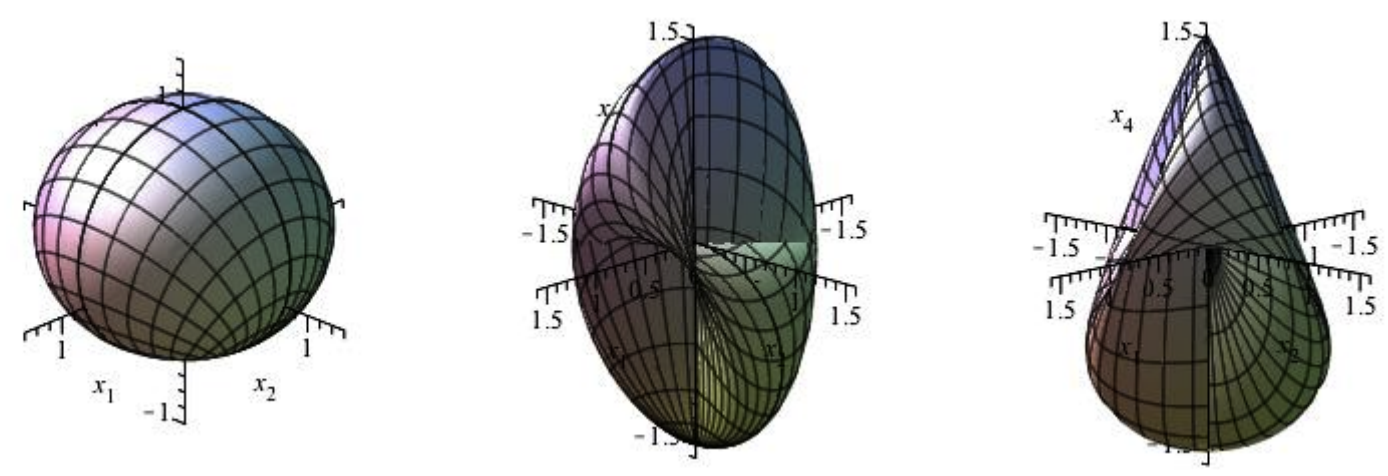

Figure 2. Projections of surface from Example 6.4 to 3-D subspaces.

\subsection{Time-like homogeneous examples}

From the adaptations of Section 5 , the matrix $\Omega=\left[\omega_{j}^{i}\right]$ of Maurer-Cartan forms on $\mathcal{F}_{3}$ may be written as

$$
\Omega=\left[\begin{array}{ccccc}
0 & 0 & 0 & 0 & 0 \\
0 & 1 & 0 & 0 & 0 \\
0 & 0 & -1 & 0 & 0 \\
0 & 0 & 0 & 2 & 0 \\
0 & 0 & 0 & 0 & -2
\end{array}\right] \alpha+\left[\begin{array}{ccccc}
0 & 0 & 1 & 0 & 0 \\
1 & h_{41}^{4} & h_{32}^{3} & h_{31}^{1} & h_{41}^{1} \\
0 & -h_{32}^{4} & h_{41}^{4} & h_{31}^{2} & h_{41}^{2} \\
0 & 1 & 0 & h_{31}^{3} & h_{41}^{3} \\
0 & 0 & 0 & h_{31}^{4} & h_{41}^{4}
\end{array}\right] \omega_{0}^{1}
$$




$$
+\left[\begin{array}{ccccc}
0 & 1 & 0 & 0 & 0 \\
0 & h_{32}^{3} & -h_{41}^{3} & h_{32}^{1} & h_{42}^{1} \\
1 & h_{41}^{4} & h_{32}^{3} & h_{31}^{1} & h_{41}^{1} \\
0 & 0 & 0 & h_{32}^{3} & h_{42}^{3} \\
0 & 0 & 1 & h_{32}^{4} & h_{42}^{4}
\end{array}\right] \omega_{0}^{2}
$$

while the structure equations (2.3) may be written as

$$
d \Omega=-\Omega \wedge \Omega
$$

Substituting (6.9) into equation (6.10) and imposing the condition that all the functions $h_{j k}^{i}$ are constant leads to a system of 23 algebraic equations for the 14 unknown constants $h_{j k}^{i}$. This system has precisely one solution, which is described in the following example.

Example 6.5. The unique solution to equation (6.10) with all $h_{j k}^{i}$ constant is

$$
\Omega=\left[\begin{array}{ccccc}
0 & 0 & 0 & 0 & 0 \\
0 & 1 & 0 & 0 & 0 \\
0 & 0 & -1 & 0 & 0 \\
0 & 0 & 0 & 2 & 0 \\
0 & 0 & 0 & 0 & -2
\end{array}\right] \alpha+\left[\begin{array}{ccccc}
0 & 0 & 1 & 0 & 0 \\
1 & 0 & 0 & 0 & 0 \\
0 & 0 & 0 & 0 & \frac{2}{3} \\
0 & 1 & 0 & 0 & 0 \\
0 & 0 & 0 & 0 & 0
\end{array}\right] \omega_{0}^{1}+\left[\begin{array}{ccccc}
0 & 1 & 0 & 0 & 0 \\
0 & 0 & 0 & \frac{2}{3} & 0 \\
1 & 0 & 0 & 0 & 0 \\
0 & 0 & 0 & 0 & 0 \\
0 & 0 & 1 & 0 & 0
\end{array}\right] \omega_{0}^{2} .
$$

Furthermore, the Gauss equation (5.11) implies that the centroaffine metric has Gauss curvature $K=-\frac{1}{3}$.

As in the space-like examples, denote the matrices in equation (6.11) by $M_{0}, M_{1}, M_{2}$, respectively, so that

$$
\Omega=M_{0} \alpha+M_{1} \omega_{0}^{1}+M_{2} \omega_{0}^{2} .
$$

Then we have

$$
\left[M_{0}, M_{1}\right]=M_{1}, \quad\left[M_{1}, M_{2}\right]=\frac{1}{3} M_{0}, \quad\left[M_{2}, M_{0}\right]=M_{2} .
$$

These bracket relations imply that the Lie algebra $\mathfrak{g} \subset \mathfrak{g l}(5, \mathbb{R})$ spanned by $\left(M_{0}, M_{1}, M_{2}\right)$ is isomorphic to $\mathfrak{s o}(2,1)$. Furthermore, it is straightforward to check that $\mathfrak{g}$ acts irreducibly on $\mathbb{R}^{5} \backslash\{0\}$. Similarly to the previous examples, $\mathfrak{s o}(2,1)$ has a unique irreducible 5-dimensional representation, and this representation arises from a (unique) irreducible representation of $\mathrm{SO}^{+}(2,1)$; it follows that the Lie group $G \subset \mathrm{GL}(5, \mathbb{R})$ corresponding to the Lie algebra $\mathfrak{g}$ is isomorphic to $\mathrm{SO}^{+}(2,1)$.

We will compute a local parametrization for $\Sigma$ more or less as in the space-like examples, by computing 1-parameter subgroups of $G$ and taking products of the resulting group elements. Unfortunately, the basis $\left(M_{0}, M_{1}, M_{2}\right)$ is not well-suited to generating a surjective parametrization, so first we need to modify it slightly. To this end, observe that a basis $\left(\bar{M}_{0}, \bar{M}_{1}, \bar{M}_{2}\right)$ for the standard representation of $\mathfrak{s o}(2,1)$ with the same bracket relations as $\left(M_{0}, \sqrt{3} M_{1}, \sqrt{3} M_{2}\right)$ is given by

$$
\bar{M}_{0}=\left[\begin{array}{ccc}
0 & 0 & 0 \\
0 & 1 & 0 \\
0 & 0 & -1
\end{array}\right], \quad \bar{M}_{1}=\left[\begin{array}{ccc}
0 & 0 & 1 \\
1 & 0 & 0 \\
0 & 0 & 0
\end{array}\right], \quad \bar{M}_{2}=\left[\begin{array}{lll}
0 & 1 & 0 \\
0 & 0 & 0 \\
1 & 0 & 0
\end{array}\right] .
$$

Now consider the modified basis

$$
\bar{M}_{0}^{\prime}=\bar{M}_{0}, \quad \bar{M}_{1}^{\prime}=\frac{1}{\sqrt{2}}\left(\bar{M}_{1}-\bar{M}_{2}\right), \quad \bar{M}_{2}^{\prime}=\frac{1}{\sqrt{2}}\left(\bar{M}_{1}+\bar{M}_{2}\right) .
$$


Exponentiating this modified basis yields the 1-parameter subgroups

$$
\begin{aligned}
& \bar{g}_{0}(t)=\left[\begin{array}{ccc}
1 & 0 & 0 \\
0 & e^{t} & 0 \\
0 & 0 & e^{-t}
\end{array}\right], \quad \bar{g}_{1}(u)=\left[\begin{array}{ccc}
\cos (u) & -\frac{1}{\sqrt{2}} \sin (u) & \frac{1}{\sqrt{2}} \sin (u) \\
\frac{1}{\sqrt{2}} \sin (u) & \frac{1}{2}(1+\cos (u)) & \frac{1}{2}(1-\cos (u)) \\
-\frac{1}{\sqrt{2}} \sin (u) & \frac{1}{2}(1-\cos (u)) & \frac{1}{2}(1+\cos (u))
\end{array}\right], \\
& \bar{g}_{2}(v)=\left[\begin{array}{ccc}
\cosh (v) & \frac{1}{\sqrt{2}} \sinh (v) & \frac{1}{\sqrt{2}} \sinh (v) \\
\frac{1}{\sqrt{2}} \sinh (v) & \frac{1}{2}(\cosh (v)+1) & \frac{1}{2}(\cosh (v)-1) \\
\frac{1}{\sqrt{2}} \sinh (v) & \frac{1}{2}(\cosh (v)-1) & \frac{1}{2}(\cosh (v)+1)
\end{array}\right] .
\end{aligned}
$$

Then the map $f: \mathbb{R}^{3} \rightarrow \mathrm{SO}^{+}(2,1)$ defined by

$$
\begin{aligned}
f(u, v, t)= & \bar{g}_{1}(u) \bar{g}_{2}(v) \bar{g}_{0}(t) \\
= & {\left[\begin{array}{ccc}
\cos (u) \cosh (v) & \frac{1}{\sqrt{2}} e^{t}(\cos (u) \sinh (v) & \frac{1}{\sqrt{2}} e^{-t}(\cos (u) \sinh (v) \\
\frac{1}{\sqrt{2}}(\sin (u) \cosh (v) & \frac{1}{2} e^{t}(\sin (u) \sinh (v)) & \frac{1}{2} e^{-t}(\sin (u) \sinh (v) \\
+\sinh (v)) & +\cosh (v)+\cos (u)) & +\cosh (v)-\cos (u)) \\
-\frac{1}{\sqrt{2}}(\sin (u) \cosh (v) & -\frac{1}{2} e^{t}(\sin (u) \sinh (v) & -\frac{1}{2} e^{-t}(\sin (u) \sinh (v) \\
-\sinh (v)) & -\cosh (v)+\cos (u)) & -\cosh (v)-\cos (u))
\end{array}\right] }
\end{aligned}
$$

is surjective onto $\mathrm{SO}^{+}(2,1)$. Thus the analogous map $f: \mathbb{R}^{3} \rightarrow G$ will be surjective onto $G$, and the map $\bar{f}=\pi \circ f$ will be surjective onto $\Sigma$.

So, define the 1-parameter subgroups

$$
g_{0}(t)=\exp \left(t M_{0}\right), \quad g_{1}(u)=\exp \left(u \sqrt{\frac{3}{2}}\left(M_{1}-M_{2}\right)\right), \quad g_{2}(v)=\exp \left(v \sqrt{\frac{3}{2}}\left(M_{1}+M_{2}\right)\right) .
$$

(The explicit expressions for these group elements are each too large to fit on one line and are not particularly enlightening.) Then set

$$
\begin{aligned}
\bar{f}(u, v, t)= & \pi\left(g_{1}(u) \cdot g_{2}(v) \cdot g_{0}(t)\right) \\
= & {\left[\begin{array}{c}
\frac{1}{4}\left[3 \cos ^{2}(u)(\cosh (2 v)+1)-2\right] \\
\frac{\sqrt{6}}{4} \cos (u)[\sin (u)(\cosh (2 v)+1)+\sinh (2 v)] \\
-\frac{\sqrt{6}}{4} \cos (u)[\sin (u)(\cosh (2 v)+1)-\sinh (2 v)] \\
-\frac{3}{8}\left[\cos ^{2}(u)(\cosh (2 v)+1)-2(\cosh (2 v)+\sin (u) \sinh (2 v))\right] \\
-\frac{3}{8}\left[\cos ^{2}(u)(\cosh (2 v)+1)-2(\cosh (2 v)-\sin (u) \sinh (2 v))\right]
\end{array}\right] . }
\end{aligned}
$$

It follows from the discussion above that $\bar{f}$ is a surjective map onto $\Sigma$, and we see that $\bar{f}$ is also independent of $t$. Moreover, it is straightforward to check that the tangent vectors $\bar{f}_{u}, \bar{f}_{v}$ are linearly independent for all $(u, v) \in \mathbb{R}^{2}$; therefore $\bar{f}$ parametrizes a smooth surface $\Sigma \subset \mathbb{R}^{5} \backslash\{0\}$, as expected.

We can compute the centroaffine metric on $\Sigma$ as in the previous examples. Let $f: \mathbb{R}^{3} \rightarrow G$ be the map corresponding to (6.12); i.e.,

$$
f(u, v, t)=g_{1}(u) \cdot g_{2}(v) \cdot g_{0}(t) .
$$

Then we have

$$
\Omega=M_{0} \alpha+M_{1} \omega_{0}^{1}+M_{2} \omega_{0}^{2}=f^{-1} d f .
$$



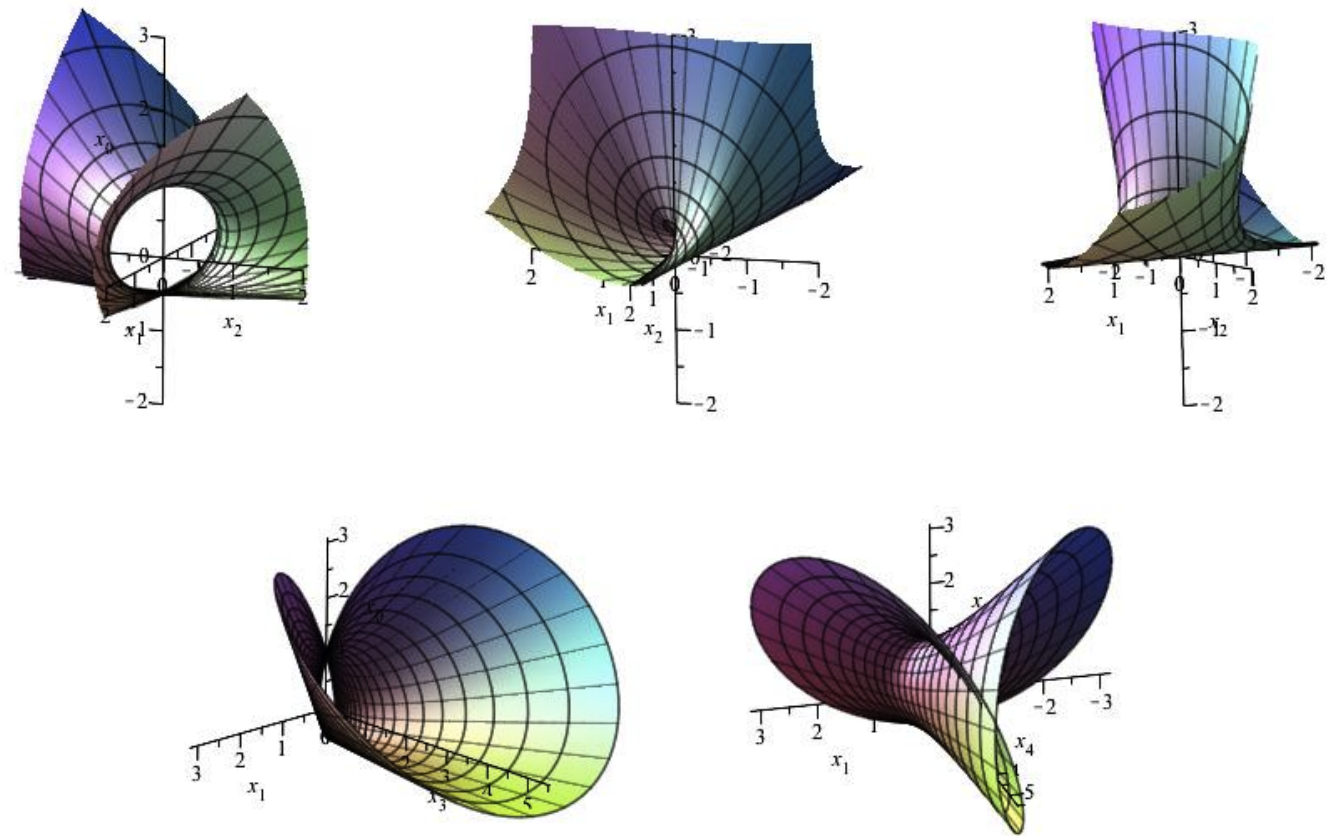

Figure 3. Projections of surface from Example 6.5 to 3-D subspaces.

Comparing these two expressions for $\Omega$ shows that

$$
\omega_{0}^{1}=\sqrt{\frac{3}{2}} e^{-t}(\cosh (v) d u+d v), \quad \omega_{0}^{2}=\sqrt{\frac{3}{2}} e^{t}(-\cosh (v) d u+d v) .
$$

Therefore, the centroaffine metric on $\Sigma$ is given by

$$
I=2 \omega_{0}^{1} \omega_{0}^{2}=3\left(-\cosh ^{2}(v) d u^{2}+d v^{2}\right) .
$$

As expected, this metric has constant Gauss curvature $K=\frac{1}{3}$.

In this case, $\mathcal{F}_{3}$ is isomorphic to the orthonormal frame bundle of the time-like surface $S^{2,1}$ consisting of all space-like vectors of length $\sqrt{3}$ in $\mathbb{R}^{2,1}$. This surface is a hyperboloid of one sheet, and so we might expect that $\Sigma$ would be diffeomorphic to this hyperboloid. However, if we regard the domain of the parametrization (6.12) as $S^{1} \times \mathbb{R}$, we see that the map (6.12) is invariant under the transformation

$$
(u, v) \rightarrow(u+\pi,-v) .
$$

Therefore, $\Sigma$ is diffeomorphic to a Möbius band, which is precisely the quotient of the hyperboloid by this map. (We note, however, that the map $f: S^{1} \times \mathbb{R} \rightarrow G$ is one-to-one, as the frame vectors $\mathbf{e}_{1}(u, v), \ldots, \mathbf{e}_{4}(u, v)$ are not preserved by the transformation (6.13).)

As in the space-like examples, we can show that $\Sigma$ is contained in the intersection of three quadric hypersurfaces in $\mathbb{R}^{5} \backslash\{0\}$. The coordinates of $\bar{f}(u, v)$ satisfy the quadratic equations

$$
3 x_{2}^{2}-x_{4}\left(4 x_{0}+2\right)=0, \quad 3 x_{1}^{2}-x_{3}\left(4 x_{0}+2\right)=0, \quad 2 x_{0}^{2}-x_{0}-3 x_{1} x_{2}-1=0 .
$$

Therefore, $\Sigma$ is contained in the (real) algebraic variety $X \subset \mathbb{R}^{5} \backslash\{0\}$ defined by equations (6.14). $\Sigma$ is an interesting and somewhat complicated subset of $X$ : first observe that the projection of $X$ to the $\left(x_{0}, x_{1}, x_{2}\right)$ coordinate 3 -plane consists of the hyperboloid of one sheet defined by 
the third equation in (6.14), minus all points of the form $\left(-\frac{1}{2}, x_{1}, x_{2}\right)$ except for $\left(-\frac{1}{2}, 0,0\right)$. The projection of $X$ to this punctured hyperboloid is one-to-one, except over the point $\left(-\frac{1}{2}, 0,0\right)$, where the inverse image consists of all points of the form $\left(-\frac{1}{2}, 0,0, x_{3}, x_{4}\right)$. Meanwhile, $\Sigma$ consists of that portion of $X$ that projects to the portion of the hyperboloid with $x_{0}>-\frac{1}{2}$, together with the curve

$$
\left\{\left(-\frac{1}{2}, 0,0, x_{3}, x_{4}\right) \mid x_{3} x_{4}=\frac{9}{4}, x_{3}, x_{4}>0\right\} .
$$

Projections of $\Sigma$ to the $\left(x_{1}, x_{2}, x_{0}\right),\left(x_{1}, x_{2}, x_{3}\right),\left(x_{1}, x_{2}, x_{4}\right),\left(x_{1}, x_{3}, x_{0}\right)$, and $\left(x_{1}, x_{4}, x_{0}\right)$ coordinate 3 -planes are shown in Fig. 3.

We collect the results of this section in the following theorem:

Theorem 6.6. Let $\bar{f}: M \rightarrow \mathbb{R}^{5} \backslash\{0\}$ be a centroaffine immersion whose image $\Sigma=\bar{f}(M)$ is a homogeneous, nondegenerate, space-like or time-like centroaffine surface. Then $\Sigma$ is equivalent via the $\mathrm{GL}(5, \mathbb{R})$-action on $\mathbb{R}^{5} \backslash\{0\}$ to one of the following:

- the immersion of the hyperbolic plane $\mathbb{H}^{2}$ of Example 6.3;

- the immersion of the sphere $S$ of Example 6.4;

- the immersion of the Lorentzian surface $S^{2,1}$ of Example 6.5.

\section{Acknowledgments}

This research was supported in part by NSF grants DMS-0908456 and DMS-1206272.

\section{References}

[1] Clelland J.N., From Frenet to Cartan: the method of moving frames, in preparation.

[2] Fulton W., Harris J., Representation theory, Graduate Texts in Mathematics, Vol. 129, Springer-Verlag, New York, 1991.

[3] Furuhata H., Minimal centroaffine immersions of codimension two, Bull. Belg. Math. Soc. Simon Stevin 7 (2000), 125-134.

[4] Furuhata H., Kurose T., Self-dual centroaffine surfaces of codimension two with constant affine mean curvature, Bull. Belg. Math. Soc. Simon Stevin 9 (2002), 573-587.

[5] Gardner R.B., Wilkens G.R., The fundamental theorems of curves and hypersurfaces in centro-affine geometry, Bull. Belg. Math. Soc. Simon Stevin 4 (1997), 379-401.

[6] Griffiths P., On Cartan's method of Lie groups and moving frames as applied to uniqueness and existence questions in differential geometry, Duke Math. J. 41 (1974), 775-814.

[7] Ivey T.A., Landsberg J.M., Cartan for beginners: differential geometry via moving frames and exterior differential systems, Graduate Studies in Mathematics, Vol. 61, Amer. Math. Soc., Providence, RI, 2003.

[8] Laugwitz D., Differentialgeometrie in Vektorräumen, unter besonderer Berücksichtigung der unendlichdimensionalen Räume, Friedr. Vieweg \& Sohn, Braunschweig, 1965.

[9] Li A.M., Wang C.P., Canonical centroaffine hypersurfaces in $\mathbb{R}^{n+1}$, Results Math. 20 (1991), 660-681.

[10] Liu H.L., Wang C.P., The centroaffine Tchebychev operator, Results Math. 27 (1995), 77-92.

[11] Mayer O., Myller A., La géométrie centroaffine des courbes planes, Ann. Scí. de l'Universit'e de Jassy 18 (1933), 234-280.

[12] Milnor J., Husemoller D., Symmetric bilinear forms, Ergebnisse der Mathematik und ihrer Grenzgebiete, Vol. 73, Springer-Verlag, New York - Heidelberg, 1973.

[13] Nomizu K., Sasaki T., Centroaffine immersions of codimension two and projective hypersurface theory, Nagoya Math. J. 132 (1993), 63-90.

[14] Nomizu K., Vrancken L., A new equiaffine theory for surfaces in $\mathbb{R}^{4}$, Internat. J. Math. 4 (1993), $127-165$. 
[15] Scharlach C., Centroaffine first order invariants of surfaces in $\mathbb{R}^{4}$, Results Math. 27 (1995), 141-159.

[16] Scharlach C., Centroaffine differential geometry of (positive) definite oriented surfaces in $\mathbb{R}^{4}$, in New Developments in Differential Geometry (Budapest, 1996), Kluwer Acad. Publ., Dordrecht, 1999, 411-428.

[17] Scharlach C., Simon U., Verstraelen L., Vrancken L., A new intrinsic curvature invariant for centroaffine hypersurfaces, Beiträge Algebra Geom. 38 (1997), 437-458.

[18] Scharlach C., Vrancken L., A curvature invariant for centroaffine hypersurfaces. II, in Geometry and Topology of Submanifolds, VIII (Brussels, 1995/Nordfjordeid, 1995), World Sci. Publ., River Edge, NJ, 1996, 341-350.

[19] Scharlach C., Vrancken L., Centroaffine surfaces in $\mathbb{R}^{4}$ with planar $\nabla$-geodesics, Proc. Amer. Math. Soc. 126 (1998), 213-219.

[20] Wang C.P., Centroaffine minimal hypersurfaces in $\mathbb{R}^{n+1}$, Geom. Dedicata 51 (1994), 63-74.

[21] Wilkens G.R., Centro-affine geometry in the plane and feedback invariants of two-state scalar control systems, in Differential Geometry and Control (Boulder, CO, 1997), Proc. Sympos. Pure Math., Vol. 64, Amer. Math. Soc., Providence, RI, 1999, 319-333.

[22] Yang Y., Liu H., Minimal centroaffine immersions of codimension two, Results Math. 52 (2008), $423-437$.

[23] Yang Y., Yu Y., Liu H., Flat centroaffine surfaces with the degenerate second fundamental form and vanishing Pick invariant in $\mathbb{R}^{4}$, J. Math. Anal. Appl. 397 (2013), 161-171. 